\title{
Tensor Decomposition of Human Narrowband Oscillatory Brain Activity in Frequency, Space and Time
}

\author{
Roman Rosipal ${ }^{1,2}$, Zuzana Roštáková ${ }^{1}$ and Leonardo Jose Trejo² \\ ${ }^{1}$ Institute of Measurement Science, Slovak Academy of Sciences \\ ${ }^{2}$ Pacific Development and Technology, LLC
}

Correspondence concerning this article should be addressed to Roman Rosipal, Institute of Measurement Science, Slovak Academy of Sciences, Dúbravská cesta 9, 84104 Bratislava, Slovakia. Email: roman.rosipal@savba.sk 


\section{Abstract}

Many brain processes in health and disease are associated with modulation of narrowband brain oscillations (NBOs) in the scalp-recorded EEG, which exhibit specific frequency spectra and scalp topography. Isolating and tracking NBOs over time using algorithms is useful in domains such as braincomputer interfaces or when measuring the EEG effects of experimental manipulations. Previously, we successfully applied modified tensor methods for identifying and tracking NBO activity over time or conditions. We introduced frequency and spatial constraints that greatly improved their physiological plausibility. In this paper we rigorously demonstrate the power and precision of tensor methods to separate, isolate and track NBOs using sources simulated with forward models. This allows us to control the attributes of NBOs and validate tensor solutions. We find that tensor methods can accurately identify, separate and track NBOs over time, using realistic sources either alone or in combination, and compare favorably to well-known spatio-spectral decomposition method for NBO estimation.

Keywords: electroencephalogram, human, EEG, oscillations, tensor decomposition, PARAFAC, Tucker decomposition 


\section{Introduction}

An important part of basic and applied EEG research is the quantitative measurement of narrowband brain oscillations (NBOs), which are characterized by stable frequency spectra and scalp topography, but which vary in amplitude over time, often appearing in sustained bursts of waves. Well known examples include the posterior rhythm or alpha waves, sleep spindles, frontal intermittent delta activity, midline frontal theta rhythms, and $\mu$ or sensorimotor rhythms over central regions. Medications may also synchronize EEG oscillations. For example, some drugs produce synchronous beta band oscillations, often waxing and waning rhythmically as if beating (Blume, 2006). Current theory states that NBOs are primarily generated by the summation of extracellular currents surrounding large numbers of synchronously firing cortical pyramidal cells (Nunez \& Srinivasan, 2006), which are associated with sensory, perceptual, cognitive and motor functions (Buzsáki \& Draguhn, 2004) and may serve as electrophysiological signatures of functional network activity, such as the default mode network (Jann, Kottlow, Dierks, Boesch, \& Koenig, 2010). Accurately measuring the activations of NBOs over time or conditions is extremely valuable for understanding brain functions and for practical and clinical applications of quantitative EEGs. Unfortunately, the measurement of NBOs is hindered by prevalent broadband cortical activity, or "background EEG." The broadband activity mimics properties of a scalefree system in which the power spectral density is inversely proportional to frequency, having a $1 / f$-like power spectrum property (He, 2014). NBO and broadband neuronal activities simultaneously propagate through the cortex, cerebrospinal fluid, skull and scalp, and contribute to the scalp-recorded EEG. Complex volume conduction properties of these layers substantially confound spatial and spectral information of simultaneously active EEG sources, making it difficult to separate them (Nunez P. L., 1981; Gloor, 1984; Winter, Nunez, Ding, \& Srinivasan, 2007).

Many algorithms have been developed and applied to quantitative measurement of NBOs (Nikulin, Nolte, \& Curio, 2011; Cohen, 2017; Srinivasan, Winter, \& Nunez, 2006; Hyvärinen, Ramkumar, 
Parkkonen, \& Hari, 2010; Hansen, et al., 2019). Spatial separation of NBOs requires multichannel EEG recordings, which are treated as multivariate data, unlike univariate methods in which each electrode is analyzed separately. Linear spatial filter techniques create a set of techniques where unfiltered or bandpassed filtered multichannel EEG data are decomposed to find a set of spatial weight vectors through which the data are projected (filtered) and time-scores ${ }^{1}$ computed. Each spatial vector is assumed to represent a unique scalp EEG pattern whose presence or absence reflects the activations of a given NBO or a mixture of NBOs. In most algorithms, spectral information is not directly used when extracting the spatial weight vectors and this information is obtained after transferring time-scores into the frequency domain. This leads to the problem that if the band-passed or unfiltered EEG data contains several NBOs, whose activation partly or fully overlaps in time and space, they may be recovered as a composite. This would limit the accuracy of tracking the activation of a single NBO over time. Widely used multivariate statistical techniques like principal component analysis (PCA) or independent component analysis (ICA) belong to this set (Rutledge \& Jouan-Rimbaud, 2013; Delorme, Palmer, Onton, Oostenveld, \& Makeig, 2012). These methods are usually applied as a blind source separation method and in general do not require pre-filtering of EEG signals into the frequency range of the targeted oscillatory sources. On the other hand, methods like spatio-spectral decomposition (SSD) (Nikulin, Nolte, \& Curio, 2011) or generalized eigendecomposition (GED) (de Cheveigné \& Arzounian, 2015) require band-passed filtered EEG data around the frequency of the extracted NBO. Cohen provided a detailed and careful comparison of these methods on simulated and real data and discussed their advantages and disadvantages (Cohen, 2017).

\footnotetext{
${ }^{1} \mathrm{~A}$ time-score is a scalar that represents the activation of single NBO or a selected set of NBOs during a specific time interval. Activation is understood as the EEG power increase at the atom's specific frequency and spatial location.
} 
Tensor decomposition represents a different set of techniques where information from three or more modalities can be combined to represent high-dimensional multivariate data in terms of a small number of multi-dimensional latent variables (Kolda \& Bader, 2009). In EEG analysis, these modalities most often represent frequency (amplitude or power spectrum), space (electrodes), and time (time-series of activations). Information and data are arranged into a three-way array (tensor). However, other modalities such as experimental conditions or treatment groups can be added to form a higher order multi-way tensor. This creates an advantage over matrix forms (such as PCA or ICA), where unfolding of selected modalities into multiple two-way representations is needed to interpret results. Tensor decomposition methods, including parallel factor analysis (PARAFAC) and Tucker decomposition, are then applied to the tensor to identify fundamental latent variables of a unique representation of the data (Bro, 1997; Tucker, 1966; Cichocki, Zdunek, Phan, \& Amari, 2009). We denote these elementary components as atoms (Miwakeichi, et al., 2004) and refer to the process as atomic decomposition of EEG (Roštáková, Rosipal, Seifpour, \& Trejo, 2020). What makes the tensor decomposition approach powerful in EEG data analysis, is not only the possibility of extracting separate time-scores (TS) and spatial and frequency weight vectors (signatures) of each atom, but also the possibility of applying constraints to manipulate their form. For example, non-negativity constraints on TS and signatures simplify the physiological interpretation of atoms, and additional constraints of unimodality or bimodality could lead us to the expected solution consisting of a single frequency characteristic for each extracted NBO or its specific scalp distribution. Interestingly, to our knowledge, in EEG research, the unimodality constraint imposed on frequency signatures (FS) has not been considered in literature so far, except in our own studies (Roštáková, Rosipal, \& Seifpour, 2020; Rosipal, et al., 2019). To extend this understanding of constraints for the tensor analysis of EEG, we propose new variants of PARAFAC and Tucker models. In addition to unimodality imposed on FS, we studied unimodal and bimodal constraints on spatial signatures (SS). Respectively, these constraints lead to single point or dipole-like distributions of the identified NBOs. To further support this 
approach, we also analyzed scalp EEG data transformed by the surface spline Laplacian (SSL) method (Nunez \& Srinivasan, 2006). That method filters out low spatial frequency components of scalp EEG and sharpens localization of NBOs otherwise hidden by the broadband component of the EEG signal.

Another interesting aspect of using tensor decomposition methods in EEG research is that the approach has not been carefully validated on simulated data where the characteristics and time activations of simulated NBOs in the data are known. Here we use precisely controlled simulated data to ensure the accuracy of the methods for identifying and measuring NBOs.

We assume the concept of scalp-recorded EEG as containing NBOs with stable spectral and spatial properties which are separable from the background broadband EEG. To separate these two components of EEG, prior to tensor decomposition, we decompose EEG into its harmonic (oscillatory) and broadband (also known as fractal) components. This is done by estimating the amplitude spectrum of the broadband component and subtracting it from the total amplitude spectrum. For this, we use irregular resampling auto-spectral analysis (IRASA) (Wen \& Liu, 2016). As expected, we have found that applying tensor decomposition only to the oscillatory spectral component leads to more accurate identification and measurement of NBOs than to the total spectrum or the broadband spectrum (Roštáková, Rosipal, Seifpour, \& Trejo, 2020; Rosipal, et al., 2019).

We follow the design of Cohen's excellent simulation study (Cohen, 2017). The activation of some 2,004 cortical sources is generated and projected onto the scalp EEG by using a forward modeling approach. Simulated data allows us to precisely control cortical position, oscillation frequency and time activation of the generated neuronal oscillations. We then add a set of up to seven NBOs, consisting of a unilateral source and three bilateral pairs, oscillating at four different frequencies. Sources associated with four frequencies may be active at different times or their activations may overlap. The anatomical distribution of sources follows the results obtained by analyzing electrocorticogram (ECoG) signals recorded during a motor imagery task (Stolk, et al., 2019), as well as our experimental work focused on 
motor neurorehabilitation of subjects with movement impairments due to a stroke (Rosipal, et al., 2019). The setting represents the challenging task of extracting several NBOs close in frequency and spatial distribution. We investigated the performance of all linear spatial filters used in (Cohen 2017), and we chose the SSD method as the best performing method to compare with tensor decomposition.

Finally, we select an example of a real multi-channel, resting-state eyes-closed, EEG where a dominant alpha rhythm is present and visually detectable. We extracted the PARAFAC atoms and compared them with the SSD solution.

\section{Material and Methods}

\subsection{Tensor models and constraints}

Consider a three-way tensor $X \in R^{I \times J \times K}$, where $/$ represents the number of time points (ordinal series of discrete times at which segments of EEG time series are sampled for analysis), $J$ is the number of electrodes (active electrodes of a referential montage), and $K$ is the number of frequencies (bins of the frequency amplitude spectrum). Tensor data in their raw form are difficult to interpret. Therefore, they are usually represented as a decomposition of lower-dimensional and easy interpretable matrices (twoway tensor). In this study we consider two tensor decomposition methods - parallel factor analysis (PARAFAC) (Harshman, 1970; Carroll \& Chang, 1970) and Tucker model (Tucker, 1966).

Both methods decompose a three-way tensor $X \in R^{I \times J \times K}$ into factor matrices $A \in R^{I \times M}$ - timescores, $B \in R^{J \times N}$ - spatial signatures, $C \in R^{K \times O}$ - frequency signatures, and a core (mixing) tensor $G \in$ $R^{M \times N \times O}$. The $(i, j, k)^{t h}$ element of $X$ can be expressed as

$$
x_{i j k}=\sum_{m=1}^{M} \sum_{n=1}^{N} \sum_{o=1}^{O} g_{m n o} a_{i m} b_{j n} c_{k o}+e_{i j k}, \quad i=1, \ldots, I ; j=1, \ldots, J ; \quad k=1, \ldots, K,
$$

where $e_{i j k}$ is the $(i, j, k)^{\text {th }}$ element of an error tensor $E \in R^{I \times J \times K}$. The unknown factor matrices and core (mixing) tensor are estimated by the alternating least squares algorithm (ALS) (Young \& de Leeuw, 1978). 
The number of factor matrix columns is user-defined and is usually set significantly lower than the number of rows; that is $M \ll I, N \ll J$ and $O \ll K$.

The main difference between the PARAFAC and Tucker models is the form of constraints applied to the mixing tensor $G$ and the number of considered hidden factors. PARAFAC factor matrices have the same number of columns $(M=N=O)$ and the core tensor follows a super-diagonal structure which means that non-zero elements are located only on the main super-diagonal of the mixing tensor $G$. Therefore, for PARAFAC, the $o^{\text {th }}$ column of $C$ is related only to the $o^{\text {th }}$ column of $B$ and only to the $o^{\text {th }}$ column of $A$ (Figure 1). These columns of individual modalities create an elementary unit of the PARAFAC decomposition - an atom. Tucker factor matrices may have different numbers of columns, which means that non-zero elements may be located on or off the super-diagonal of the mixing tensor G. Therefore, in the Tucker model we create the atom corresponding to the $o^{\text {th }}$ column of $C$ by linearly combining a subset of $A$ and $B$ columns (Figure 2, bottom). The coefficients of this linear combination are stored in the mixing tensor $G$.

To improve the interpretability and stability of the solution, we apply realistic constraints to the columns of factor matrices or the mixing tensor. These constraints usually take the form of orthogonality, nonnegativity or unimodality (Kiers \& Smilde, 1998). The orthogonality constraint usually reduces computational time of ALS but limits the generality of the solution. As we describe in Section 2.3, our input data represent the non-negative oscillatory part of the amplitude spectrum, therefore non-negativity seems to be a natural constraint in all three modes, that is time scores (TS), spatial signature (SS) and frequency signature (FS). For a given atom, any positive TS value then represents its activation, which is the product of frequencies, weighted by the positive values of the atom's FS, at the spatial locations weighted by the positive values of the atom's SS. We design our PARAFAC and Tucker models to isolate and measure NBOs. Therefore, we constrain FS to be unimodal, so as to obtain a single spectral peak in the FS. This allows us to identify atoms with unimodal narrow frequency bands and consequently separate 
their activations in space and time. However, we stress the fact that we are not restricted to this reductionist approach. If our aim is to track atoms that combine frequency bands, TS of unimodal atoms can be combined into a single time series of activations. For example, if a source exhibits fundamental and harmonic peaks with the same SS, the TS of each harmonic component can be combined to track the activation of the composite over time. An example of where this occurs in nature is in the auditory steadystate response, or ASSR, where fundamental and harmonics combine to form the integrated response (Brenner, et al., 2009). Finally, in addition to non-negativity of SS, we validated unimodality and bimodality constraints in the spatial modality. This is motivated by the fact that NBOs may be modeled by synchronous dipoles, which may be unilateral or bilateral and may have radial or tangential orientations. Note, that the inherently non-negative amplitude spectrum should render the SS of any NBO as a set of non-negative values but without non-negativity constraints negative values may appear in the tensor decomposition. With the non-negativity constraint, we do not see negative values in the SS. Such combination of unimodality, bimodality and non-negativity constraints have not been considered in EEG literature prior to our applications. In addition, we also had to adapt tensor decomposition methods to compute solutions with the bimodality constraint.

In our previous study (Roštáková, Rosipal, Seifpour, \& Trejo, 2020), we observed that the Tucker model with the unconstrained structure of the mixing tensor faced numerical problems and did not properly detect the latent structure of data. On the other hand, we observed a substantial improvement when the mixing tensor was constrained to have a non-negative structure. Together with the nonnegativity constraints applied to factor matrices this defines the non-negative Tucker decomposition (NTD) method (Cichocki, Zdunek, Phan, \& Amari, 2009).

For implementation of both PARAFAC and Tucker models, we used version 3.31 of the N-way toolbox (Andersson \& Bro, 2000) of MATLAB (The MathWorks, Inc.). The toolbox is freely available online 
at http://www.models.life.ku.dk/nwaytoolbox. For the bimodality constraint, we used proprietary MATLAB code available upon request.

\subsection{Simulated data}

We applied an anatomical forward model consisting of 2,004 dipoles placed in gray matter. This model was used in a comparative study of linear spatial filters for identifying oscillatory activity (Cohen, 2017) and was obtained from the authors' on-line repository (http://mikexcohen.com/data/, downloaded, June 20, 2020). The model was computed using the Brainstorm toolbox (Tadel, Baillet, Mosher, Pantazis, \& Leahy, 2011) in MATLAB.

We modeled narrowband oscillatory sources with seven dipoles at four different frequencies 1) a dipole located at the frontal midline region mimicking a $\theta$ rhythm at $5 \mathrm{~Hz}, 2$ ) left and right hemisphere dipoles located within the somatosensory cortex representing a $\mu$ rhythm at $8 \mathrm{~Hz}, 3$ ) left and right hemisphere dipoles located within the somatomotor cortex representing a $\beta$ rhythm at $14 \mathrm{~Hz}$, and 4) left and right hemisphere dipoles located within the posterior cortex representing a visual $\alpha$ rhythm at $11 \mathrm{~Hz}$

(Figure 3). Each oscillation was generated as a sinusoidal signal with a random modulation of frequency and amplitude at each time point. This was done by using normally distributed random noise in the frequency-domain convolved with a Gaussian kernel (Cohen, 2017). The peak frequency and full-width at half-maximum of this filter was set to $15 \mathrm{~Hz}$. A different gain was applied to signals of the four oscillation frequencies, to mimic the power spectrum proportions of these oscillations observed in real EEG data records (Figure 3). Sixty seconds of a continuous signal of each oscillation frequency were generated at the sampling frequency of $256 \mathrm{~Hz}$. These time series were then convolved with a Hann window of the length of 5 to $10 \mathrm{sec}$. The length and time position of the window was randomly selected for each of the four different simulated frequencies, but the window was same for the left and right hemisphere sources of the same frequency. We considered scenarios where windows for each of the four different frequencies are either overlapped or not overlapped in time. 
A fractional Brownian motion process with the Hurst parameter set to 0.6 was used to simulate background brain activity at each of the 2,004 dipoles. The process closely mimics a characteristic scalefree property of broadband brain activity, also manifested by a $1 / f$-like power spectrum (He, 2014). The generated traces were multiplied by two different gain constants, which yielded different signal-to-noise ratios (SNR) for the simulated narrow-band oscillations and broadband activity (see the last paragraph of this section and Table 1). These two gains were selected to create sets with a low and high signal-to-noise ratios (SNR) between an oscillations and broadband activity. We denote these two sets as SNR low $_{\text {and }}$ $S N R_{\text {high, }}$, respectively.

After setting the activation at all dipoles, the forward model was applied and EEG time series at 64 electrodes arranged according to the 10-20 system were generated. This was done separately for every SNR setting.

A second variant of EEG data was constructed by applying a spline surface Laplacian (SSL) transformation to the generated scalp EEG (Nunez, et al., 1994), (http://ssltool.sourceforge.net/, Version v1.5). A realistic head surface model given by a triangular mesh was used. We denote this transformed scalp EEG data as SSL-EEG. The idea of the transformation is to enhance shallow oscillatory peaks, otherwise hidden in the signal generated by the broadband brain activity.

Specific SNR values were computed using the scalp EEG data. This was done separately for every frequency and separately for EEG and SSL-EEG datasets. First, EEG data without broadband brain activity were generated to determine scalp locations with maximum power. Data were transformed into frequency domain and EEG channels with maximum values for each of the seven oscillatory sources were identified (see topographic maps in Figure 3). At these EEG channels, separately for the SNR low $_{\text {and }}$ SNR $R_{\text {high }}$ datasets, the ratio between the power spectrum of the oscillation frequency and the mean power spectrum of frequencies below $(-1$ to $-2 \mathrm{~Hz})$ and above $(+1$ to $+2 \mathrm{~Hz})$ the given oscillation frequency was computed. Ten times the $\log _{10}$ of the computed ratio was used to set the final SNR value for the given 
oscillation frequency. The same procedure was carried out separately for SSL-EEG signals. Table 1 summarizes all computed SNR values. Note, for lateralized NBOs, that is $\mu, \alpha$ and $\beta$, we report the average SNR value of the left and right hemisphere when the whole scalp EEG signals are decomposed.

Data are freely available at http://aiolos.um.savba.sk/ roman/rrLab/datacodes.html

\subsection{Real EEG data}

To demonstrate the principle and validity of the tensor decomposition methods on real EEG data, we selected one participant's 62-sec long record of 64-channel EEG. Data were recorded during the reststate eyes-closed condition and were part of a broader neurofeedback-oriented study (Trejo, Rosipal, \& Nunez, 2009). A BioSemi EEG Recording System (Biosemi B.V.) with a sampling frequency of $512 \mathrm{~Hz}$ was used. The pre-processing step includes re-referencing using the linked mastoid reference, band-pass filtering into the range of $1 \mathrm{~Hz}$ to $35 \mathrm{~Hz}$ and down-sampling to $256 \mathrm{~Hz}$ (with a non-causal anti-aliasing filter cutoff at $128 \mathrm{~Hz}$ ). Visual data inspection shows seven detectable bursts of a $10.5 \mathrm{~Hz}$ alpha rhythm, dominant at the occipital scalp region and also detected at a subset of frontal and central EEG electrodes. Due to the strong $10.5 \mathrm{~Hz}$ rhythm at the Iz (inion) EEG channel, this channel at the boundary of the SSL model was removed before applying SSL and not used in the analysis. The whole segment represents a very stable and clean EEG recording, a few visually detectable artifact periods were removed. This resulted in 56-sec of 63-channel artifact-free EEG data used for the analysis, which was carried out in the same way as for the simulated EEG records.

\subsection{IRASA}

Simulated scalp EEG records were split into overlapping 2-s long segments (898.4 ms overlap) with a sliding step size of $101.6 \mathrm{~ms}$ ( 26 time points). This yielded 571 segments for a total of 59.89 -s of simulated scalp EEG data. For each 2-s segment we applied the IRASA method (Wen \& Liu, 2016). IRASA decomposes the amplitude spectrum of each segment into a fractal (scale-free) and an oscillatory part. The oscillatory and fractal components of EEG may be generated by different mechanisms, so it is important to estimate 
them separately, especially when the focus of the measurement is on localized NBOs, as is the case here (Buzsáki \& Draguhn, 2004; He, 2014). The oscillatory part of the amplitude spectrum was obtained by subtracting the fractal part from the total spectrum estimate. Negative values of the oscillatory spectrum were set to zero and the transformation $10 * \log _{10}(x+1)$ was applied. Transformed oscillatory spectrum data were arranged into a three-way tensor $X \in R^{I \times J \times K}$, as discussed in Section 2.1, where $I=571$ represents the number of all two-second time windows, $J=64$ is the number of electrodes, and $K=43$ is the number of frequencies selected. In this study we consider only the frequency range between 4 and $25 \mathrm{~Hz}$ with a step size of $0.5 \mathrm{~Hz}$. Lower bands $(<4 \mathrm{~Hz})$ were excluded as this would need to be treated by considering longer segments needed to estimate slow oscillations. Higher bands (> $25 \mathrm{~Hz}$ ) were excluded due to the practical limits of IRASA to adequately separate oscillatory and fractal components for these faster frequencies. This is associated with the standard EEG problem of correctly estimating higher frequencies due to their amplitude reduction related to increasing frequency.

\subsection{Spatio-spectral decomposition (SSD)}

In the literature, linear spatial filters are often used for multichannel EEG signal analysis. A detailed and thorough comparison of linear spatial filters was carried out on simulated and real data (Cohen, 2017). Following Cohen's results, we decided to compare the spatio-spectral decomposition (SSD) method (Nikulin, Nolte, \& Curio, 2011) with the proposed tensor decomposition methods.

First, a spatial covariance matrix is computed from the band-pass filtered EEG, where the bandpass is set to include the frequency of the NBO we wish to recover. Similarly, the second covariance matrix is constructed using the filtered EEG signal at neighboring "flanking" frequencies (noise), which derive from the band-pass filtered EEG above and below the neuronal oscillation frequency. The SSD then solves the generalized eigen-decomposition problem, where one matrix is the noise covariance matrix, and the second matrix represents the sum of the signal covariance and noise covariance matrices. Solving the eigen-decomposition problem will maximize the power in the frequency of the studied oscillation and 
minimize the power at the neighboring frequencies. The set of eigenvectors belonging to the largest eigenvalues represents spatial filters with maximal separation (Cohen, 2017). The time-scores can then be obtained by applying the spatial filters to the multichannel EEG data. The frequency characteristic (signature) of the spatial filter can be estimated by transferring time-score values into a frequency domain representation. For this purpose, we applied the IRASA method and extracted the oscillatory part of the power spectrum. To be consistent with the non-negative TS of the PARAFAC and Tucker models, we computed and report absolute values of the SSD time-scores.

The SSD method requires a prior information about the frequency of the NBO one wants to recover. If this is known, the method operates reasonably well (Cohen, 2017). However, this is often not the case in practice, especially if we aim to detect less dominant or sporadically occurring rhythms which are hard to specify in advance. Other problems may arise if more than one oscillatory activity occurs within the selected frequency range. Moreover, these rhythms can be active at different times, spatial locations as well as be close in frequency. Although, we did not aim to study these aspects of SSD, we also report on a few properties of SSD we observed when compared to the focused tensor decomposition methods.

We focus on a whole frequency range into which our target frequencies fall rather than on a single specific frequency. Therefore, the EEG signal was band-pass filtered by a $2^{\text {nd }}$ order Butterworth filter in the frequency range of 4 to $15 \mathrm{~Hz}$. This represents the filtered signal including the four studied oscillations. The "flanking" frequency interval was set $2 \mathrm{~Hz}$ below and above this range and the same type of bandpass filter was used.

\subsection{Model order and selection criterion}

With decreasing SNR, the recovery of the rhythms of interest deteriorates. A higher proportion of noise usually requires increasing the order of the tensor models to detect less dominant rhythms whose power approaches the noise level. We varied the PARAFAC model order between 4 to 15 and consider the 
Tucker model with 4 to 8 factors varied in each mode. In the case of the SSD model, we investigated the first 15 extracted components.

Because we aim to show that tensor decomposition can isolate and accurately measure NBOs hidden in the background EEG, we need a criterion by which the best solution of each tensor model can be chosen. We designed the following criterion, which we denote Crit, reflecting maximum separability between the activation and deactivation time of each oscillation. For every single tensor decomposition solution, we compute the mean value of the estimated TS over the time interval with nonzero activation. This value is computed for every oscillation frequency separately. Next, we compute the percentage of TS exceeding the computed mean during the time when a given oscillation is not active. We transform this percentage value into the interval of 0 to 1 to get the final value of Crit. In other words, Crit=1 means that we can clearly identify EEG segments from times when the detected NBO is activated. On the other hand, Crit $=0.5$ means that the number of TS inside and outside of the activation interval which exceeds the computed mean is same, and the time of activation of the targeted NBO can't be correctly detected from TS.

We applied the same principle in the case of the SSD method, where Crit is computed using absolute values of the estimated TS.

\section{Results}

\subsection{Tensor models and SSD}

For reference, we compared the SSD and tensor methods on both $\mathrm{SNR}_{\text {high }}$ and $\mathrm{SNR}$ low sets. Four NBOs were activated with no overlap between Hann windows, which determine the cortical time activation of each NBO. In Figure 4A, results of SSD are depicted for the case of a higher SNR between oscillatory cortical signals and cortical background activity. We can observe good separation of $\theta(5 \mathrm{~Hz}), \mu$ $(8 \mathrm{~Hz})$, and $\alpha(11 \mathrm{~Hz})$ oscillations. For $\beta(14 \mathrm{~Hz})$, the performance degrades, and we can observe that components consisting of a mixture of NBOs are present. This may represent a problem in practice 
because the activation of individual oscillations becomes challenging to assign unambiguously. This is even harder to do when oscillations overlap in time. We observed a small change when SSD was applied to SSLEEG, that is, EEG transformed by the surface spline Laplacian method. Decreasing SNR, that is using the

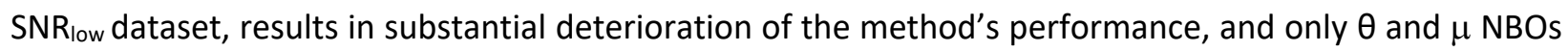
were partially detectable (Figure 4B). Again, we observed no considerable difference when SSD was

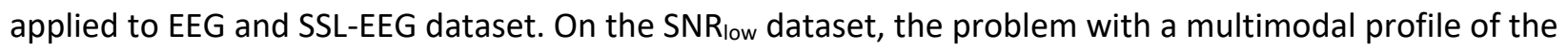
power spectral density (PSD) persists. Note that the PSD profiles represent an oscillatory part of the SSD time-scores spectrum computed by the IRASA method. Like Cohen's results (Cohen 2017), we observed superior and consistent SSD results compared to GED, ICA, and PCA methods.

Focusing our attention on the tensor decomposition methods, in Figure 5A, PARAFAC results for the EEG dataset with $S N R_{\text {high }}$ are depicted. We can see a clear separation of all NBOs but $\theta$. In general, we observed a lower PARAFAC method's ability to detect $\theta$ oscillations when EEG data without SSL transformation were used. This is due to a higher level of the cortical background activity in the slower frequency range which spreads over all scalp locations. However, after applying a unimodal spatial constraint, the $\theta$ NBO detection improved. In particular, we observed a more vital improvement after limiting the solution to EEG electrodes covering only the midline and right hemisphere scalp locations (Figure 5B). However, and very importantly, we observed a significant improvement in detection of all NBOs on the SSL-transformed EEG (Figure 5C). Application of PARAFAC to EEG with SNR impaired detection of NBOs, but we can still detect $\mu, \alpha$, and $\beta$ oscillations (Figure $6 \mathbf{A}$ ). However, unambiguous time activation can only be determined for the $\mu$ and $\beta$ NBOs. We also observed significant improvement in the detection of all NBOs when applying the SSL transformation to the SNR low $_{\text {dataset }}$ (Figure 6B). It should be noted that at this SNR level, the SSD method is no longer able to detect any oscillations unambiguously, and the SSL transformation does not lead to the improvement of SSD (Figure 4B). 


\subsection{Spatial constraints}

In previous examples of the tensor decomposition, we could see FS's unimodality, represented by non-zero weights in the narrow band around the peak frequency. This is an essential feature of the decomposition that allows unambiguous monitoring of the activation of each individual NBO over time. In our previous work with real EEG data, we have successfully used the unimodality constraint when searching for FS of NBOs (Rosipal, et al., 2019). Now, we extend this by applying unimodal and bimodal constraints on the spatial solution of the decomposition. The idea behind these constraints is restricting the decomposition to solutions where the detection of radial and tangential cortical sources would be enhanced. While in the case of radial cortical sources, activation of a single scalp location can be expected, tangential sources would lead to the activation of two spatially separated scalp locations. Note that in the case of EEG spectra, this activation is of the same sign, while in the case of EEG time-series, it is a location with alternating positive and negative signs, i.e., a dipole (see Figure 3).

We have already demonstrated the effect of SS unimodality in the example of Figure 5B. Focusing on the local spatial solution, we have improved the ability of PARAFAC to detect the radial source of $\theta$. To the standard non-negativity constraint, we also added a bimodality constraint on SS. Simultaneously, we investigated the effect of using EEG electrodes of the left and right hemispheres only. The PARAFAC solution for SNR low $_{\text {and }}$ SSL-EEG dataset is shown in Figure 7. We focused on the $\mu$ and $\beta$ NBOs, whose cortical sources are oriented in that they create a bimodal distribution in each hemisphere separately. The figure shows that the bimodality constraint leads to the expected clearer spatial localization of oscillations. Obviously, a priori knowledge is required to use such a pre-selected constraint. Here we used the knowledge about the lateralization of the $\mu$ and $\beta$ rhythms associated with the process of motor imagery (Stolk, et al., 2019). The example demonstrates that incorporating such an assumption may improve the solution. 


\subsection{Activation overlap}

Another critical factor in detecting NBOs is the ability of the tensor methods to detect different NBOs whose activations overlap in time. For example, in real EEG recordings, we can expect a time overlap of $\mu$ and $\beta$ which represents an interplay during the process of real or motor imagery movement. We have generated a dataset where the simulated cortical oscillations overlap in time. This overlap was set to be more extensive between the pairs of $\theta$ and $\alpha$, and $\mu$ and $\beta$ (Figure 8). We considered two situations i) where the activation of overlapping NBOs occurs only once during the $60 \mathrm{sec}$ long sequence ii) where it occurs twice. For SNR high, we observed precise detection of all simulated NBOs using the EEG as well as SSL-EEG datasets. Results for the $\mathrm{SNR}_{\text {low }}$ case and SSL-EEG dataset are depicted in Figure 8. We can see precise detection of all NBOs, except for $\theta$, which is somewhat less clear. However, by increasing the number of activation periods to two (Figure $8 \mathbf{B}$ ), we can observe improved performance and unambiguous detection of all NBOs. This is an essential point indicating that it is appropriate for the tensor decomposition methods to maintain a sufficient balance between the number of samples representing each condition. Results for the EEG dataset are similar to those obtained in the case of non-overlapping activation of NBOs. That is a good detection of NBOs up to $\theta$. The results were again improved by adding the second activation period.

\subsection{Atom tracking}

A critical aspect of NBOs measurement methods is the ability to use estimated vectors of spatial and frequency signatures to determine the activation of NBOs on data that has not been used for the decomposition. For example, when we need to monitor activation and deactivation of the oscillations in real-time or when analyzing completely new test EEG measurements. Also, we often try to reduce the set of EEG electrodes following the results obtained on high-density multichannel EEG records. Using the structure of SS, we can reduce the set of EEG electrodes only to places represented by high spatial weights. Such an approach may lead to minimal performance deterioration of classification or tracking algorithms 
with reduced electrode density (Wallerius, Trejo, Matthews, Rosipal, \& Caldwell, 2005). Also, when using a smaller number of electrodes, we cannot transform EEG data using SSL, but we may still use the obtained SSL-EEG solution to determine the placement of the reduced set of electrodes.

To validate applicability of the tensor decomposition solution on new data, we generated independent training and test datasets. Test data were generated using a new sample of the brain background activity and consist of four activation periods within the $60-$ sec long session. NBO activations within each of the four periods overlap (Figure 9). We also generated a new sample of each cortical oscillation to keep a difference between data used to estimate the model and testing data. As a training model, we used the PARAFAC model estimated on the SSL-EEG dataset with SNR scores of the test dataset computed as a projection to the training PARAFAC model are depicted in Figure 9. For the non-Laplacian EEG dataset, we can see a good ability to detect NBOs, except for $\theta$. Recall that the training model was estimated by using SSL-EEG data. Time-scores for the SSL-EEG dataset show apparent and unambiguous detection of $\mu, \alpha$, and $\beta$ rhythms. The detection of $\theta$ is less specific, but still, the activation of $\theta$ can be correctly determined in three out of the four simulated periods. By generating new samples, we reduced the SNR even more, and in the case of SSL-EEG, we were still able to detect $\mu$, $\alpha$, and $\beta$ oscillations well enough.

\subsection{The Tucker model}

In all previously reported results, we ran the NTD version of the Tucker model in parallel with PARAFAC. We found a good match between the PARAFC and NTD solutions. This opens the question of when to use a more complicated Tucker model. One of the first differences and advantages of the Tucker model is the possibility of a more compact representation. An example is the case when some modalities of NBOs overlap, whether of temporal activation, frequency or spatial distribution of atoms. We investigated the dataset with the overlap of NBO time activations similar to Figure 8B. In this example, the $\alpha$ and $\theta$ rhythms are simultaneously active over a wide interval, while the $\mu$ and $\beta$ rhythms are 
simultaneously activated with $a \approx 1$-s delay after $\alpha$ and $\theta$. Therefore, we applied the $(2,4,4)$-NTD model ${ }^{2}$ assuming a pattern of two general TS profiles. In Figure 10A we can see the precise estimate of all four FS and SS and the expected two TS profiles. Moreover, the mixing tensor $G \in \mathrm{R}_{+}^{2 \times 4 \times 4}$ (a non-negative tensor of order $2 \times 4 \times 4)$, depicted in Figure 10B, follows a sparse structure allowing us to quickly identify the only non-zero SS for a given combination of TS and FS as a linear combination of primary SS (pSS). In contrast, PARAFAC in this case required model orders of 4 or 5 for the $\mu, \alpha$ and $\beta$ rhythms and up to the order of 15 for $\theta$. With decreasing SNR, the orders of both models increase; however, the NTD model was still able to describe the data structure with fewer components than PARAFAC.

\subsection{Real EEG}

A burst of alpha rhythm followed by the period without visually detectable alpha oscillation is depicted in Figure 11A. We identified seven similar 2-3 sec long bursts of alpha during the whole 56-sec long resting-state eyes-closed EEG recording. We applied the IRASA method, with the same setting as in simulated data, to the entire $56 \mathrm{sec}$ long recording and estimated total and oscillatory parts of the power spectrum for every 2-sec long epoch. After averaging power spectrum parts through all epochs, we plot scalp topographic at $10.5 \mathrm{~Hz}$; that is, at the frequency where the maximum power in the alpha frequency range was observed (Figure 11B, C). Topographic maps indicate strong $10.5 \mathrm{~Hz}$ dominant at the occipital PO8 site and show the presence at the left occipital and frontal-central left and right scalp regions. We hypothesize that the presence of the rhythm at the more anterior areas can be due to the volume conduction effect, but to confirm this goes beyond the scope of this paper. In the next step, we applied the same procedure on data transformed with SSL. The resulting constraint of the $10.5 \mathrm{~Hz}$ signal to the right occipital region aligns with the hypothesis (Figure 11B).

\footnotetext{
${ }^{2}$ The $(2,4,4)$ notation denotes the NTD model with two time-score components, and four spatial and frequency signatures.
} 
First, we ran SSD on raw EEG and SSL transformed data. Two dominant components whose timescores matched seven alpha bursts were observed in the case of raw EEG and one in the case of SSL transformed data. To match the time-scores with the power of the $10.5 \mathrm{~Hz}$ alpha rhythm, we band-pass filtered EEG data at PO7 and PO8. We used the bandpass.m routine of MATLAB, with the Wpass parameter set to the range 9.5 to $11.5 \mathrm{~Hz}$.

For raw EEG (Figure 12A), two extracted SSD components and two PARAFAC atoms represent the left and right posterior activation centered at $\mathrm{PO} 7$ and $\mathrm{PO} 8$ scalp locations. The right hemisphere activation dominates. Time scores of the first SSD component and the PARAFAC atom can discriminate seven bursts of the alpha rhythm. We can see that the time-scores of the second SSD component (left hemisphere activation) shows a little ability to distinguish individual alpha bursts. We can also observe that the activation in the anterior regions is detected only by PARAFAC (Figure 12A, atom 1). Further inspection of the additional SSD components identified the component with the activation over the frontal-central region. However, the oscillatory power spectrum of this component consisted of several different frequency peaks (the dominant peak at $10.5 \mathrm{~Hz}$ ). Still, the time-scores of this SSD component did not discriminate the alpha bursts.

Both SSD and PARAFAC, applied to SSL transformed EEG extracted a single component reflecting the dominant $10.5 \mathrm{~Hz}$ activation in the right posterior hemisphere (Figure 12B). This closely matches the expected dominant rhythm and its spatial distribution seen in Figure 11.

\section{Discussion}

With the growing body of evidence on the importance of narrowband oscillations in the field of human brain electrophysiology, the need for their accurate isolation and measurement from scalp EEG recordings is important for tracking the effects of various treatments or manipulations over time. Spatial filtering methods represent one of a set of algorithms that are often used for this purpose (Cohen, 2017). Often the goal is not only the detection of oscillating sources but also their continuous monitoring. An 
example is their use in the field of brain-computer interface (BCl) protocols (Lotte, et al., 2018) or mental state estimation (Trejo, Kubitz, Rosipal, Kochavi, \& Montgomery, 2015). One of the limitations of spatial filtering methods is their inability to directly incorporate frequency information into their solution. The frequency information is often obtained by a separate power spectral analysis of the time scores of the extracted component. This limitation often leads to the need to filter EEG data into frequency bands containing the expected NBOs. The approach brings several pitfalls. One of them is the lack of accurate a priori knowledge about these frequencies, which may not be identifiable in the power spectrum of the recorded EEG data. The use of generally defined EEG frequency bands, such as 8 to $12 \mathrm{~Hz}$ for $\alpha$ rhythm, is very treacherous and ignores substantial individual differences related to age and other conditions. For example, using directly measured cortical activity during a motor imagery process revealed significant variation of the $\mu$ rhythm among subjects (Stolk, et al., 2019). This narrow band activity was in the range of 3 to $7 \mathrm{~Hz}$ in the most extreme case and up to the 8 to $11 \mathrm{~Hz}$ range, as generally expected for the $\mu$ rhythm, in the other case. A similar substantial inter-subject variability of the frequency ranges was observed for the $\beta$ sensorimotor oscillation associated with the same motor imagery process. Another example may be the often-observed presence of posterior $\alpha$ activity in EEG measurements on electrodes covering more anterior regions, for example the sensorimotor cortical area. In this case, the frequency peak of $\alpha$ and the motor related $\mu$ rhythm can be very close to each other. Such an inability to spatially separate EEG rhythms generated by remote cortical areas is the result of the well-known volume conduction problem (Nunez \& Srinivasan, 2006). A real example is a robust presence of the posterior $\alpha$ rhythm on the EEG electrodes covering the sensorimotor area of a patient with a cortical lesion in the left hemisphere (Rosipal, et al., 2019). In this case, we detected the $\alpha$ peak at $9.25 \mathrm{~Hz}$ and the $\mu$ rhythm peak at $7.75 \mathrm{~Hz}$, effectively creating only a $1.5 \mathrm{~Hz}$ difference between the peaks. It is not only challenging to apply such narrowband pre-filtering of the EEG signal, but above all to determine the appropriate frequency ranges of these filters. The often used $\alpha$ range of 8 to $12 \mathrm{~Hz}$ or lower $\alpha$ range of 8 to $10 \mathrm{~Hz}$, 
would essentially lead to filtering out a large part of these two specific NBOs. A partial solution to mitigate these problems is using the filter-bank approach and to search for an answer in each filter bank (Ang, Chin, Zhang, \& Guan, 2008). However, the filter-bank technique is often applied only in the case of supervised learning, such as building a classifier. However, with the problem of determination and clear neurophysiological interpretation of all present NBOs, such an approach will also encounter the problems mentioned above.

Although our primary goal was a thorough analysis of simulated data, we also performed a simple limited validation of the fundamental properties of tensor decomposition using real EEG data from a resting-state eyes-closed condition, where the detection of discrete alpha bursts was visually detectable. We found that, as with simulated data, the PARAFAC approach was highly effective at isolating the spatial and spectral profile of the alpha bursts and tracking their activations accurately over time.

We find that tensor decomposition is a powerful and elegant method for detection and monitoring NBOs. Using the tensor structure, we can represent the modalities of frequency, space and time separately. This allows us to introduce a priori information about the expected form of each modality individually. We incorporate this information into the tensor decomposition in the form of constraints. It is interesting that, for example, limiting the frequency modality to a unimodal form has not been more systematically used in the EEG literature, apart from our previous work (Roštáková, Rosipal, Seifpour, \& Trejo, 2020). We believe that this simple modification of the tensor decomposition solution leads to an elegant estimate of NBOs with unambiguous frequency patterns. This facilitates not only the isolation of NBOs but also their unambiguous time activation, because each oscillation can be tracked separately. On the other hand, if the goal is to monitor the activation and an interplay of multiple rhythms, we can easily combine FS and SS of different atoms and create a time-scores composite. For example, suppose we observe multiple NBOs in the beta frequency range (Rosipal, et al., 2019). In that case, we can still consider 
their combination and monitor activation throughout the whole beta range, not just the activation of individual NBOs.

In this article, for the first time, we also dealt in more detail with the possibility of using unimodal and bimodal restrictions on the form of the SS. We have shown that this allows accurate and spatially more precise detection of radial and tangential sources of cortical oscillations. The approach also allows for reducing EEG spatial information to a small number of areas, EEG electrodes, on the surface of the head. This is an important factor for the application of the tensor solutions when the continuous monitoring of the activation and deactivation of selected NBOs is the focus, for example, in the case of building BCl protocols (Rosipal, Porubcová, Cimrová, \& Farkaš, 2018).

We observed superior performance of tensor decompositions when applied to EEG data transformed using the surface spline Laplacian. Such a transformation results mainly in the change of the spatial distribution of NBOs but is less significant in changing the temporal variation of the oscillating sources in traces of EEG themselves. This explains the SSD method's almost negligible changes when applied to the SSL-EEG dataset and compared to the EEG dataset. In contrast, the reduction of spatial information after the SSL transformation facilitates finding a compact SS solution of the tensor decomposition. Importantly, as we have shown in the example (Figure 9), a tensor solution obtained from SSL-EEG dataset makes it possible to compute time-scores of new EEG test data not transformed via SSL. However, further quantitative study needs to be carried out to validate this step.

The tensor decomposition approach used in the article incorporates the IRASA method which separates the oscillatory component of the EEG power spectrum. This allows us to focus only on the oscillatory sources of the power spectrum and leads to a more accurate and more straightforward estimation of NBOs. We tested the approach and confirmed its usefulness and validity on real data representing EEG rhythms of motor activation and deactivation (Rosipal, et al., 2019; Rosipal, Porubcová, Cimrová, \& Farkaš, 2018). 
There are other exciting elements of the tensor decomposition. For example, the elimination of unwanted solutions masking the solutions we are interested in. We believe this interesting element is often unused when tensor methods are applied to EEG data analysis. The principle consists of the initial estimation of the tensor solution and the subsequent subtraction of the information of unwanted solutions from the initial tensor representation. We can call it the deflation step. In the second stage, we work with a tensor representation free of unwanted solutions. The procedure proved to be useful in the exploratory analysis of EEG signals for lateralized attention network function (Trejo, Rosipal, \& Nunez, 2009). Another interesting feature of the tensor methods is the possibility of incorporating a larger number of modalities and analysis of multi-way tensor data structures. For example, we have shown that incorporating a session modality leads to the estimation of general NBOs through EEG measurements recorded during multiple days (Roštáková, Rosipal, \& Seifpour, 2020). Several other types of modalities were used in the literature (Mørup, Hansen, Herrmann, Parnas, \& Arnfred, 2006).

Out of the two tensor decomposition methods-PARAFAC and the Tucker model - the usage of PARAFAC dominates in the literature, mainly due to a simpler model parameter setting and the interpretation following the principle of the same number of components in each modality. In the Tucker model, the factor matrices are related through the mixing tensor, and the interpretation of the results requires more thorough inspection, especially when the mixing tensor is dense. When the mixing tensor is sparse, the Tucker model can provide more detailed information about the data structure than PARAFAC. We found the NTD version of the Tucker model suitable for EEG data analysis and better at facilitating interpretation of the obtained solutions (Roštáková, Rosipal, Seifpour, \& Trejo, 2020). The Tucker model may provide a parsimonious representation of data when compared with PARAFAC. One of the pitfalls of PARAFAC is the degenerate solution. This is the case if data don't follow an exact multilinear, in our case trilinear, structure (Paatero, 2000; Mørup, Hansen, Arnfred, Lim, \& Madsen, 2008). This may lead to the extraction of highly correlated factors. In our setting, an analogy of a degenerate array 
(Paatero, 2000), may be the case where several NBOs share the same time activation or their spatial distributions and frequency peaks are close. It is the Tucker model which allows better matching data of such a structure and avoiding degenerate solutions.

In this study, we tried to find the best possible solution for either PARAFAC or NTD models, focusing on precise time detection of NBOs we used the Crit for this purpose (Section 2.5). Using TS, we designed the criterion intending to find the solution for each NBOs, such that maximal separation between the activation and deactivation of a given NBO is achieved. However, it must be noted that when real EEG data are analyzed, identification of NBOs from a set of decomposed candidate atoms is much harder task (Roštáková \& Rosipal, 2021). This is especially true for NBOs with a low SNR between their amplitude and background noise. Therefore, we recommend applying the following clustering approach for real experimental data analysis (Roštáková, Rosipal, Seifpour, \& Trejo, 2020). Because it is difficult in advance to estimate a correct order of the PARAFAC model or the number of components in each modality of the Tucker model, we run several PARAFAC or Tucker models with varying order and collect all results of the decomposition-atoms. In the second step, the atoms from all considered models are assigned into clusters according to the similarity between their TS, SS and FS. This reduces the number of solutions and allows us to focus on dominant clusters, which represent typical atoms systematically extracted from models of different order. The final atoms can be then represented as the cluster means in each modality. For the clustering step, we have a good experience with the nonparametric density-based clustering (DBSCAN) (Ester, Kriegel, Sander, \& Xu, 1996), the method we used in (Roštáková, Rosipal, Seifpour, \& Trejo, 2020). Naturally, the final and important step is to put these final extracted atoms into the context of the underlying experiment, cognitive or mental state of the subject, etc., and in this way to validate their relevance.

One limitation of tensor methods as proposed here is that they cannot be used to estimate traveling EEG waves. The field of traveling waves in EEG has been developing and will no doubt play an 
important future role in basic and clinical electrophysiology (Nunez P. L., 2014; Zhang, Watrous, Patel, \& Jacobs, 2018). But the tensor methods as formulated here require a fixed spatial distribution of scalp potentials associated with NESOs. This is also a limitation of other spatial decomposition methods, such as ICA or SSD.

In this study, we focused on the qualitative side of the detection of NBOs using tensor methods. For this purpose, we used simulated data where we can precisely control and evaluate the obtained solutions. Interestingly, despite the more than 15 years of use of tensor methods in the field of EEG data analysis, the validation of the approach on simulated data has not been systematically studied. Such an analysis provides a lot of essential knowledge about the tensor methods properties necessary for application to real EEG data. Systematic analysis and detailed quantitative validation and comparison of the tensor decomposition and comparisons with existing methods are a large part of our ongoing research.

\section{Acknowledgement}

Dr. Paul Nunez contributed ideas, and helped with valuable discussions of the models, results and interpretation. R.R. and Z.R. were supported by the Slovak Research and Development Agency (grant APVV-16-0202) and the VEGA grant 2/0081/19. Pacific Development and Technology LLC recorded, processed and furnished the real resting state EEG data and supported Dr. Trejo's role in the writing and editing of the manuscript. 


\section{References}

Andersson, C., \& Bro, R. (2000). The N-way Toolbox for MATLAB. Chemometrics \& Intelligent Laboratory Systems, 52(1), 1-4. doi:10.1016/S0169-7439(00)00071-X

Ang, K., Chin, Z. Y., Zhang, H., \& Guan, C. (2008). Filter Bank Common Spatial Pattern (FBCSP) in BrainComputer Interface. Proceedings of the International Joint Conference on Neural Networks, (pp. 2390-2397. doi:10.1109/IJCNN.2008.4634130).

Blume, W. T. (2006). Drug Effects on EEG. Journal of Clinical Neurophysiology, 23(4), 306-311. doi:10.1097/01.wnp.0000229137.94384.fa

Brenner, C. A., Krishnan, G. P., Vohs, J. L., Ahn, W. -Y., Hetrick, W. P., Morzorati, S. L., \& O'Donnell, B. F. (2009). Steady State Responses: Electrophysiological Assessment of Sensory Function in Schizophrenia. Schizophrenia Bulletin, 35(6), 1065-1077. doi:10.1093/schbul/sbp091

Brenner, C. A., Krishnan, G. P., Vohs, J. L., Ahn, W. -Y., Hetrick, W. P., Morzorati, S. L., \& O'Donnell, B. F. (2009). Steady State Responses: Electrophysiological Assessment of Sensory Function in Schizophrenia. Schizophrenia Bulletin, 35(6), 1065-1077. doi:10.1093/schbul/sbp091

Bro, R. (1997). PARAFAC. Tutorial and applications. Chemometrics and Intelligent Laboratory Systems, 38(2), 149-171. doi:10.1016/S0169-7439(97)00032-4.

Buzsáki, G., \& Draguhn, A. (2004). Neuronal oscillations in cortical networks. Science, 304(5679), 19261929. doi:10.1126/science.1099745.

Carroll, J. D., \& Chang, J.-J. (1970). Analysis of individual differences in multidimensional scaling via an Nway generalization of "Eckart-Young" decomposition. Psychometrika, 35(3), 283-319. doi:10.1007/BF02310791. 
Cichocki, A., Zdunek, R., Phan, A. H., \& Amari, S.-I. (2009). Nonnegative Matrix and Tensor Factorizations: Applications to Exploratory Multi-Way Data Analysis and Blind Source Separation. John Wiley \& Sons. doi:10.1002/9780470747278.

Cohen, M. X. (2017). Comparison of linear spatial filters for identifying oscillatory activity in multichannel data. Journal of Neuroscience Methods, 1-12. doi:doi.org/10.1016/j.jneumeth.2016.12.016.

de Cheveigné, A., \& Arzounian, D. (2015). Scanning for oscillations. Journal of Neural Engineering, 12, 066020. doi:10.1088/1741-2560/12/6/066020.

Delorme, A., Palmer, J., Onton, J., Oostenveld, R., \& Makeig, S. (2012). Independent EEG sources are dipolar. PLoS One, 7, e30135. doi:doi.org/10.1371/journal.pone.0030135.

Ester, M., Kriegel, H.-P., Sander, J., \& Xu, X. (1996). A density-based algorithm for discovering clusters in large spatial databases with noise. Second International Conference on Knowledge Discovery \& Data Mining (pp. 226-231). American Association for Artificial Intelligence. doi:dl.acm.org/doi/10.5555/3001460.3001507.

Gloor, P. (1984). Neuronal generators and the problem of localization in electroencephalography: application of volume conductor theory to electroencephalography. Journal of Clinical Neurophysiology, 2(4), 327-354. doi:10.1097/00004691-198510000-00002.

Hansen, S. T., Hemakom, A., Safeldt, M. G., Krohne, L. K., Madsen, K. H., Siebner, H. R., ... Hansen, L. K. (2019). Unmixing Oscillatory Brain Activity by EEG Source Localization and Empirical Mode Decomposition. Computational Intelligence and Neuroscience, ID 5618303. doi:10.1155/2019/5618303.

Harshman, R. (1970). Foundations of the PARAFAC procedure: models and conditions for an "explanatory" multimodal factor analysis. UCLA Working Papers in Phonetics, 16(1), 1-84.

He, B. J. (2014). Scale-free brain activity: past, present and future. Trends in Cognitive Sciences, 8(9), 480-487. doi:10.1016/j.tics.2014.04.003. 
Hyvärinen, A., Ramkumar, P., Parkkonen, L., \& Hari, R. (2010). Independent component analysis of shorttime Fourier transforms for spontaneous EEG/MEG analysis. Neurolmage, 49(1), 257-271. doi:10.1016/j.neuroimage.2009.08.028.

Jann, K., Kottlow, M., Dierks, T., Boesch, C., \& Koenig, T. (2010). Topographic electrophysiological signatures of FMRI Resting State Networks. . PloS one, 5(9), e12945.

Kiers, H. A., \& Smilde, A. K. (1998). Constrained three-mode factor analysis as a tool for parameter estimation with second-order instrumental data. Journal of Chemometrics, 12(2), 125-147. doi:10.1002/(SICI)1099-128X(199803/04)12:2<125::AID-CEM504>3.0.CO;2-D.

Kolda, T., \& Bader, B. W. (2009). Tensor Decompositions and Applications. SIAM Review, 51(3), 455-500. doi:10.1137/07070111X.

Lotte, F., Bougrain, L., Cichocki, A., Clerc, M., Congedo, M., Rakotomamonjy, A., \& Yger, F. (2018). A review of classification algorithms for EEG-based brain-computer interfaces: a 10 year update. Journal of Neural Engineering, 15(3), 031005 (28pp). doi:10.1088/1741-2552/aab2f2.

Mørup, M., Hansen, L. K., Arnfred, S. M., Lim, L.-H., \& Madsen, K. H. (2008). Shift-invariant multilinear decomposition of neuroimaging data. Neurolmage, 42, 1439-1450. doi:10.1016/j.neuroimage.2008.05.062.

Mørup, M., Hansen, L. K., Herrmann, C. S., Parnas, J., \& Arnfred, S. (2006). Parallel Factor Analysis as an exploratory tool for wavelet transformed event-related EEG. Neurolmage, 29(3), 938-947. doi:doi.org/10.1016/j.neuroimage.2005.08.005.

Martínez-Cagigal, V. (2020). Topographic EEG/MEG plot. Retrieved from MATLAB Central File Exchange: https://www.mathworks.com/matlabcentral/fileexchange/72729-topographic-eeg-meg-plot Miwakeichi, F., Martínez-Montes, E., Valdés-Sosa, P. A., Nishiyama, N., Mizuhara, H., \& Yamaguchia, Y. (2004). Decomposing EEG data into space-time-frequency components using Parallel Factor Analysis. Neurolmage, 22, 1035-1045. doi:10.1016/j.neuroimage.2004.03.039. 
Nikulin, V. V., Nolte, G., \& Curio, G. (2011). A novel method for reliable and fast extraction of neuronal EEG/MEG oscillations on the basis of spatio-spectral decomposition. Neurolmage, 55(4), 15281535. doi:10.1016/j.neuroimage.2011.01.057.

Nunez, P. L. (1981). A Study of Origins of the Time Dependencies of Scalp EEG: I - Theoretical Basis. IEEE Transactions on Biomedical Engineering, 28(3), 271-280. doi:10.1109/tbme.1981.324700.

Nunez, P. L. (2014). Neocortical dynamics due to axon propagation delays in cortico-cortical fibers: EEG traveling and standing waves with implications for top-down influences on local networks and white matter disease. Brain Research, 1542, 138-166.

Nunez, P. L., \& Srinivasan, R. (2006). Electric Fields of the Brain: The Neurophysics of EEG. Oxford University Press. doi:10.1093/acprof:oso/9780195050387.001.0001.

Nunez, P. L., Silberstein, R. B., Cadusch, P. J., Wijesinghe, R. S., Westdorp, A. F., \& Srinivasan, R. (1994). A theoretical and experimental study of high resolution EEG based on surface Laplacians and cortical imaging. Electroencephalography and Clinical Neurophysiology, 90(1), 40-57. doi:10.1016/0013-4694(94)90112-0.

Paatero, P. (2000). Construction and analysis of degenerate PARAFAC models. Journal of Chemometrics, 14, 285-299. doi:10.1002/1099-128X(200005/06)14:3<285::AID-CEM584>3.0.CO;2-1.

Roštáková, Z., \& Rosipal, R. (2021). Determining the number of components in the PARAFAC model with a nonnegative tensor structure: A simulated EEG data study. Retrieved from PsyArXiv Preprints: https://psyarxiv.com/8r3h2

Roštáková, Z., Rosipal, R., \& Seifpour, S. (2020). Tucker Tensor Decomposition of Multi-session EEG Data. In I. Farkaš, P. Masulli, \& S. Wermter (Ed.), Artificial Neural Networks and Machine Learning, Lecture Notes in Computer Science, Springer, 12396, s. 115-126. doi:10.1007/978-3-030-616090_10. 
Roštáková, Z., Rosipal, R., Seifpour, S., \& Trejo, L. J. (2020). A comparison of non-negative Tucker decomposition and parallel factor analysis for identification and measurement of human EEG rhythms. Measurement science review, 20(3), 126-138. doi:10.2478/msr-2020-0015.

Rosipal, R., Porubcová, N., Barančok, P., Cimrova, B., Farkaš, I., \& Trejo, L. J. (2019). Effects of Mirror-Box Therapy on Modulation of Sensorimotor EEG Oscillatory Rhythms: A Single-Case Longitudinal Study. Journal of Neurophysiology, 121(2), 620-633. doi:10.1152/jn.00599.2018.

Rosipal, R., Porubcová, N., Cimrová, B., \& Farkaš, I. (2018). Mirror-therapy as a way to start BCl robotassisted rehabilitation: a single case longitudinal study of a patient with hemiparesis. poster presented at The Seventh International BCI Meeting. Pacific Grove, CA, USA.

Rosipal, R., Trejo, L. J., Roštáková, Z., \& Cimrová, B. (2018). Atomic Decomposition of Human EEG Oscillations In Medical Research And Pharmaceutical Trials. Neuropsychobiolgy, 77(3), 135-136. doi:10.1159/000496817.

Rutledge, D. N., \& Jouan-Rimbaud, B. (2013). Independent components analysis with the JADE algorithm. Trends in Analytical Chemistry, 50, 22-32. doi:10.1016/j.trac.2013.03.013.

Srinivasan, R., Winter, W. R., \& Nunez, P. L. (2006). Source analysis of EEG oscillations using highresolution EEG and MEG. Progress in Brain Research, 159, 29-42. doi:10.1016/S00796123(06)59003-X.

Stolk, A., Brinkman, L., Vansteensel, M. J., Aarnoutse, E., Leijten, F. S., Dijkerman, C. H., ... Toni, I. (2019). Electrocorticographic dissociation of alpha and beta rhythmic activity in the human sensorimotor system. eLife, 8, e48065. doi:10.7554/eLife.48065.001.

Tadel, F., Baillet, S., Mosher, J., Pantazis, D., \& Leahy, R. (2011). Brainstorm: a user-friendly application for MEG/EEG analysis. Computational Intelligence and Neuroscience, ID 879716. doi:10.1155/2011/879716. 
Trejo, L. J., Kubitz, K., Rosipal, R., Kochavi, R. L., \& Montgomery, L. D. (2015). EEG-based estimation and classification of mental fatigue. Psychology, 6(5), 572-589. doi:10.4236/psych.2015.65055

Trejo, L. J., Rosipal, R., \& Nunez, P. L. (2009). Advanced Physiological Estimation of Cognitive Status (APECS). Final project report, U.S. Army Research Office, Research Triangle Park, NC.

Tucker, L. R. (1966). Some mathematical notes on three-mode factor analysis. Psychometrika, 31(3), 279-311. doi:10.1007/BF02289464.

Wallerius, J., Trejo, L., Matthews, R., Rosipal, R., \& Caldwell, J. (2005). Robust feature extraction and classification of EEG spectra for real-time classification of cognitive state. Proceedings of the 11th International Conference on Human Computer Interaction (pp. 302-311. doi:10.1007/978-3540-73216-7_16). Las Vegas, Nevada: Lawrence Erlbaum Associates.

Wen, H., \& Liu, Z. (2016). Separating fractal and oscillatory components in the power spectrum of neurophysi- ological signal. Brain Topography, 29(1), 13-26. doi:10.1007/s10548-015-0448-0.

Winter, W. R., Nunez, P. L., Ding, J., \& Srinivasan, R. (2007). Comparison of the effect of volume conduction on EEG coherence with the effect of field spread on MEG coherence. Statistics in Medicine, 26, 3946-3957. doi:10.1002/sim.2978.

Young, F. T., \& de Leeuw, J. (1978). The principal components of mixed measurement level multivariate data: An alternating least squares method with optimal scaling features. Psychometrika, 43, 279281. doi:10.1007/BF02293871.

Zhang, H., Watrous, A. J., Patel, A., \& Jacobs, J. (2018). Theta and Alpha Oscillations Are Traveling Waves in the Human Neocortex. Neuron, 98(6), 1269-1281. 


\section{Figures and Tables}

Figure 1: Scheme of PARAFAC model. A 3-way tensor of time-space-frequency data representation is decomposed into a series of atoms each represented by time-scores (A), spatial signatures (B) and frequency signatures $(C)$. The scheme depicts the solution with 4 atoms (components), which represents the case $M=N=0=4$ of eg. (1).

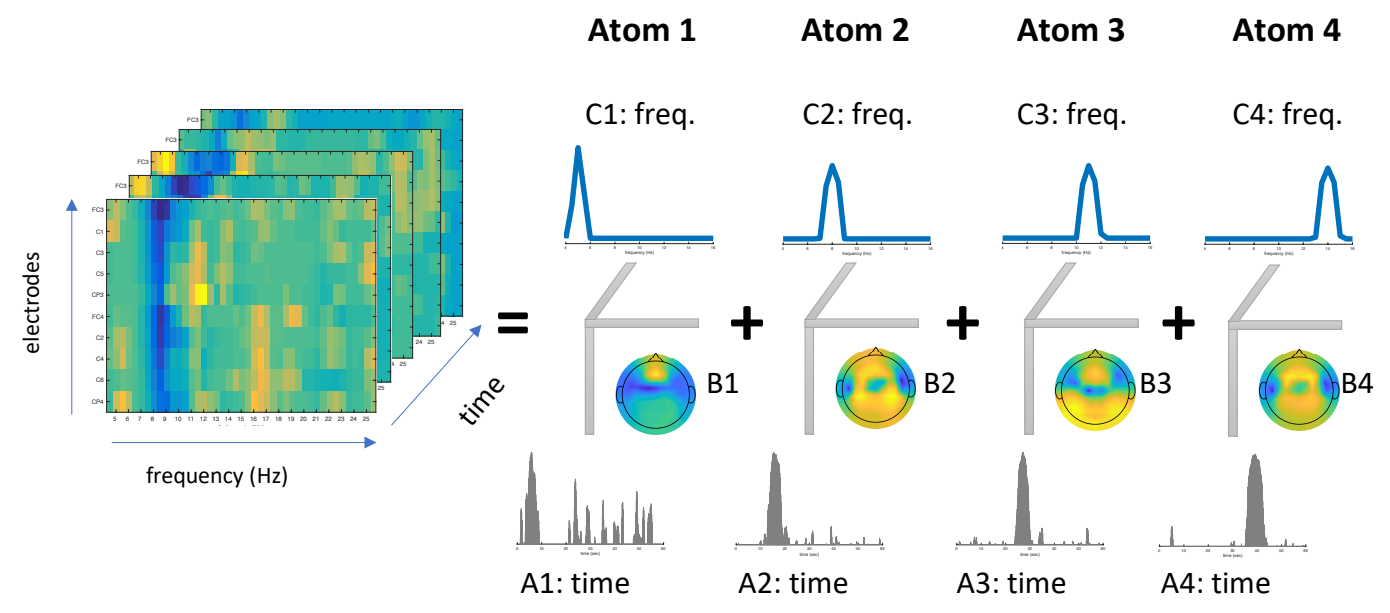


Figure 2: Scheme of the Tucker model. A 3-way tensor of time-space-frequency data representation is decomposed into a series of time-scores ( $A)$, primary spatial signatures $(B)$ and frequency signatures (C). The scheme depicts the Tucker model with 5 time-scores, 4 spatial signatures and 6 frequency signature; $M=5, N=4, O=6$ of eq. (1). Using the mixing matrix $G$, three atoms with specific frequency signatures are constructed using the extracted time-scores and spatial signatures.

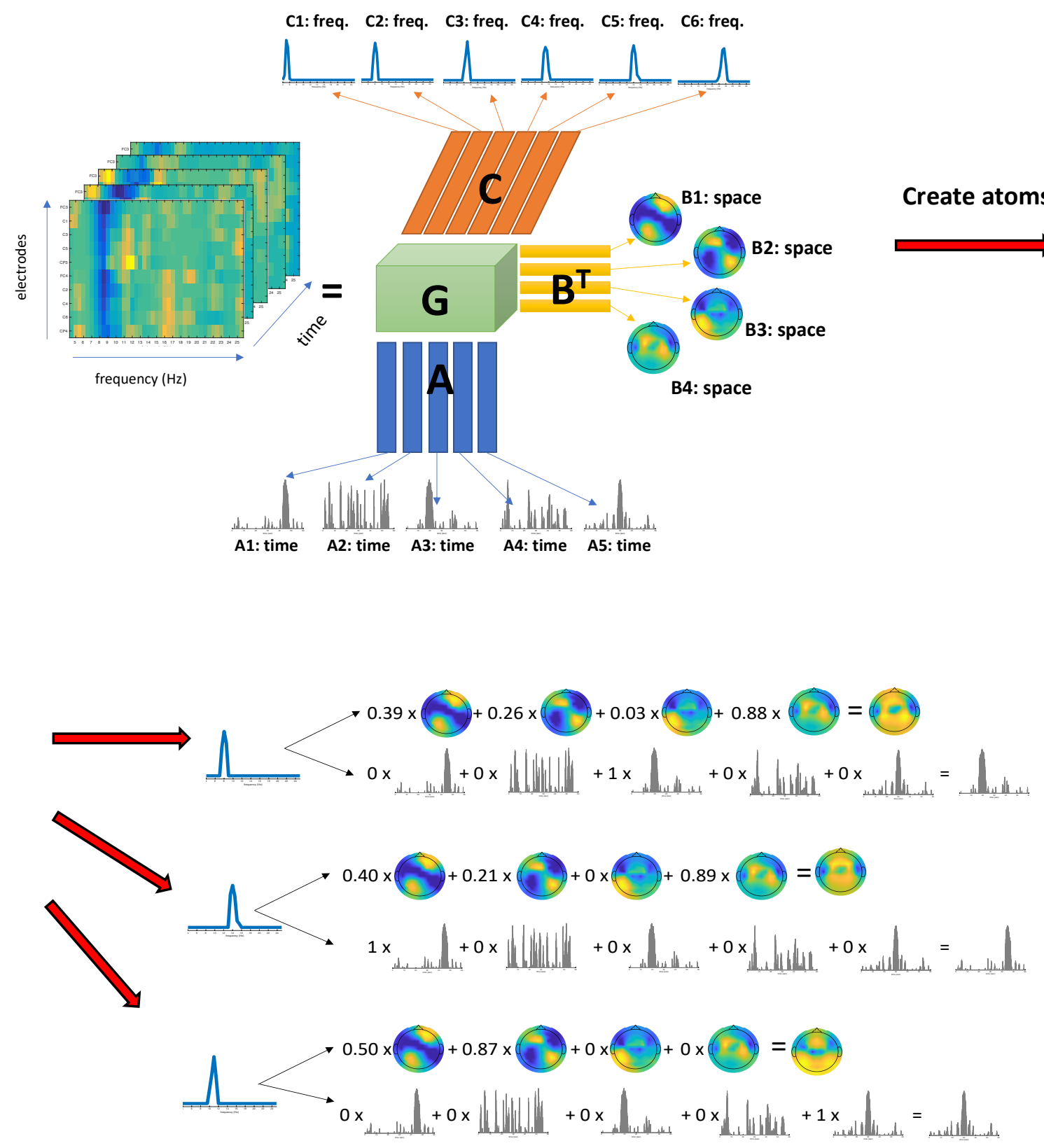

Atom 1

Atom 2

Atom 3 
Figure 3: Simulated narrowband brain oscillation (NBOs) without background brain activity. A) Locations and orientations of the NBOs cortical sources, red $-5 \mathrm{~Hz} \theta$, cyan and yellow $-14 \mathrm{~Hz} \beta$, green and magenta $-8 \mathrm{~Hz} \mu$, blue and black $-11 \mathrm{~Hz} \alpha$. B) First row: topographic maps of selected EEG spectral band amplitudes. Second row: topographic maps of the surface spline Laplacian transformed EEG (SSL-EEG). Third row: time-based topographic maps of selected EEG spectral band amplitudes taken at the maximal amplitude activation +0.4 sec of each NBO. Fourth row: same as the third row but for the SSL-EEG. C) Left: An example of the $5 \mathrm{~Hz}$ oscillations convolved with the Hann window (red curve). Right: Activation of the $5 \mathrm{~Hz}$ oscillation used for the forward modeling. D) Power spectrum density at two selected EEG electrodes for the EEG and SSL-EEG. Topographic maps were created using public-domain Topographic EEG/MEG MATLAB plot functions (Martínez-Cagigal, 2020).

A)
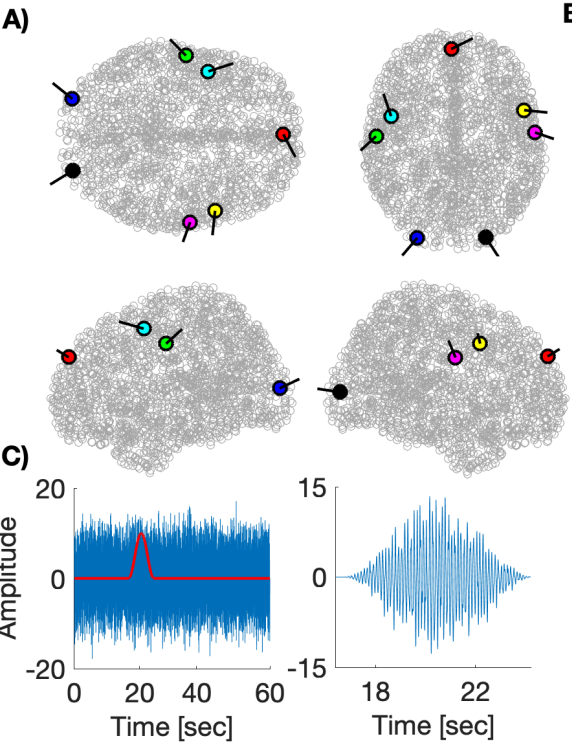

D)

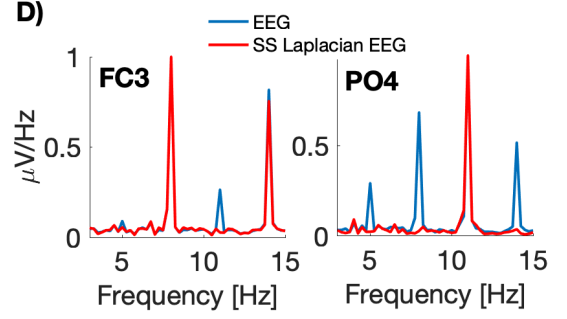

B)

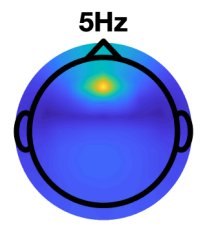

$5 \mathrm{~Hz}$
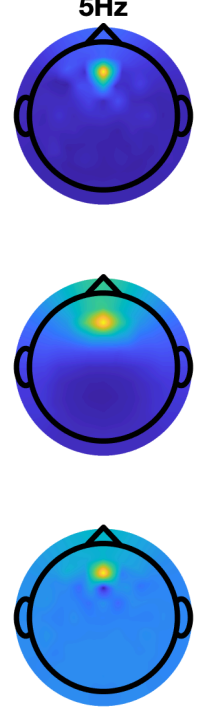

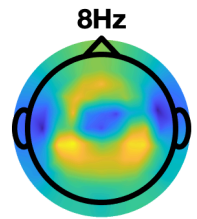

$8 \mathrm{~Hz}$
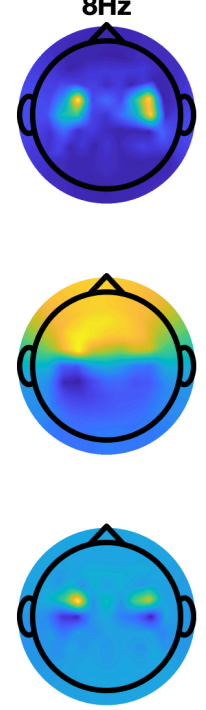
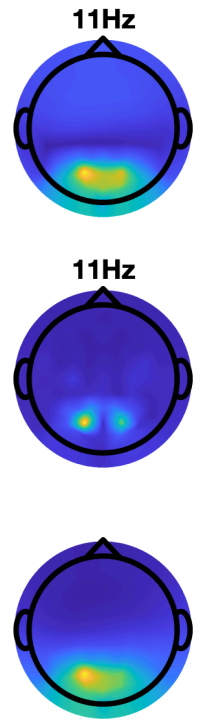

$11 \mathrm{~Hz}$
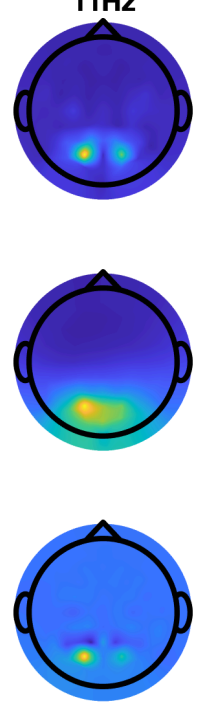

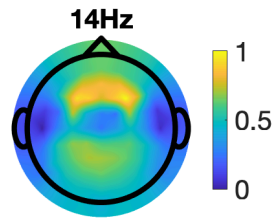

$14 \mathrm{~Hz}$
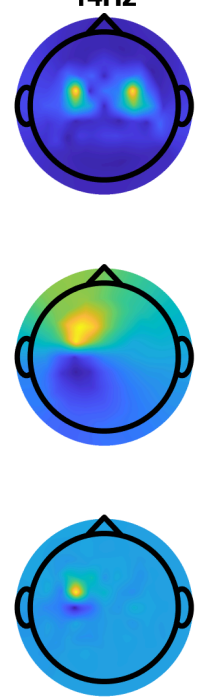
Figure 4: SSD results. Each column represents absolute time-scores, spatial signature and frequency signature of each NBO. The frequency signature of the SSD components is represented by the oscillatory part of the corresponding time-scores power spectrum. Red Hann window plots overlayed with time-scores represent time activation of the oscillation and black Hann windows activation of remaining NBOs. Dashed horizontal represents the threshold used to compute values of Crit (see section Error! Reference source not found.). A) SSD for the EEG dataset with SNR high. B) SSD for the SSL-EEG dataset with SNR $R_{\text {low. }}$

A)
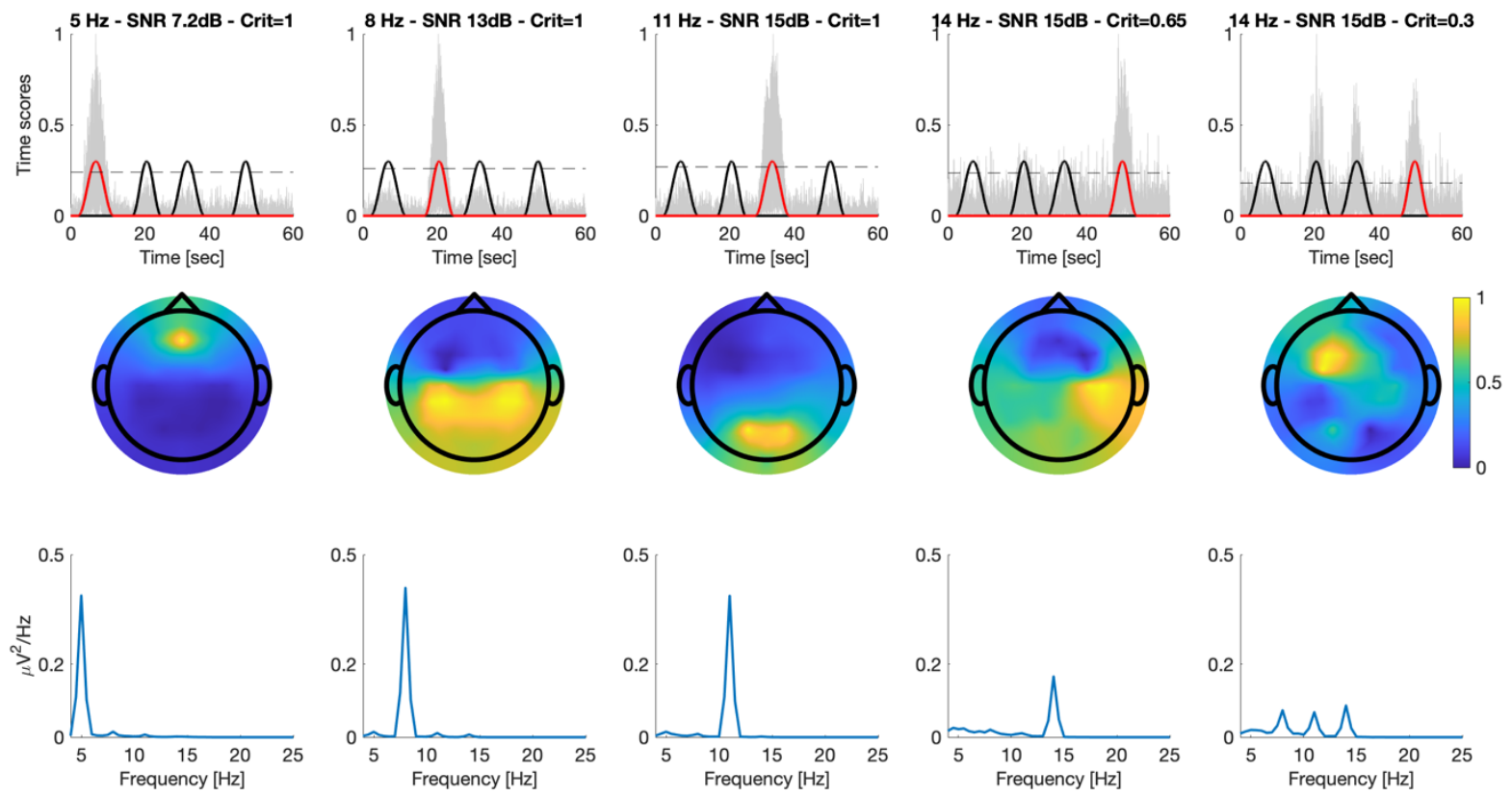

B)
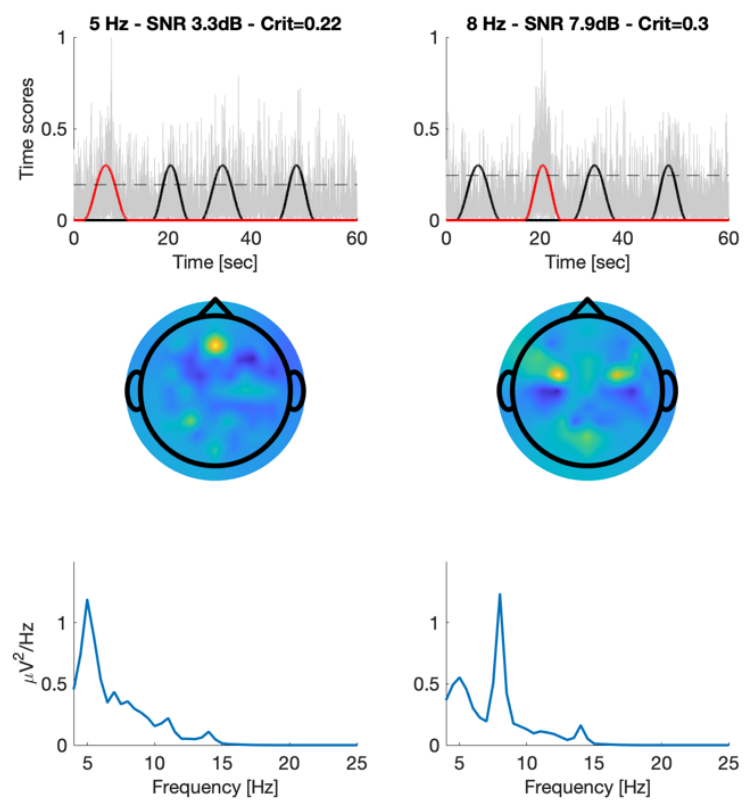
Figure 5: PARAFAC results. Description of plots same as in Figure 4. Time-scores of PARAFAC are constraint to nonnegative values. A) PARAFAC for the EEG dataset with SNR high. B) $\theta N B O$. PARAFAC for the EEG dataset with $S N R_{\text {high }}$ and with unimodality spatial constraint and right hemisphere electrodes used. $C$ ) PARAFAC for the SSL-EEG dataset with SNR high.

A)
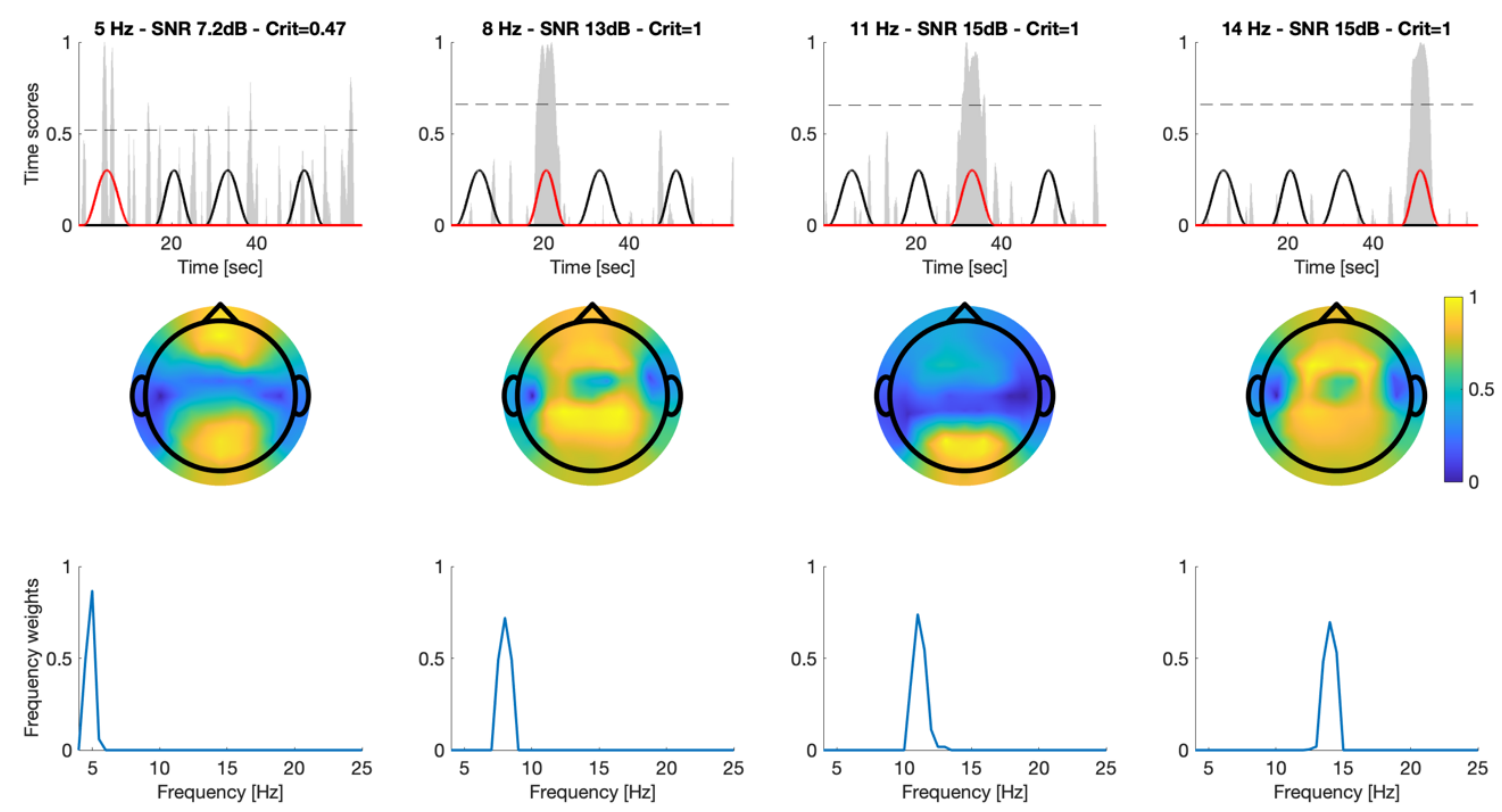

B)
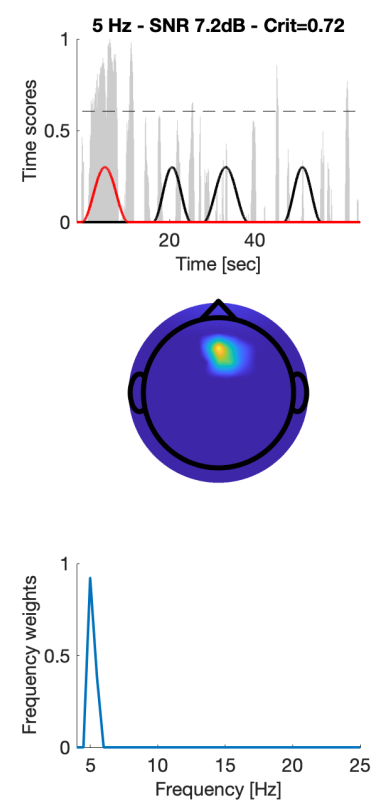
C)
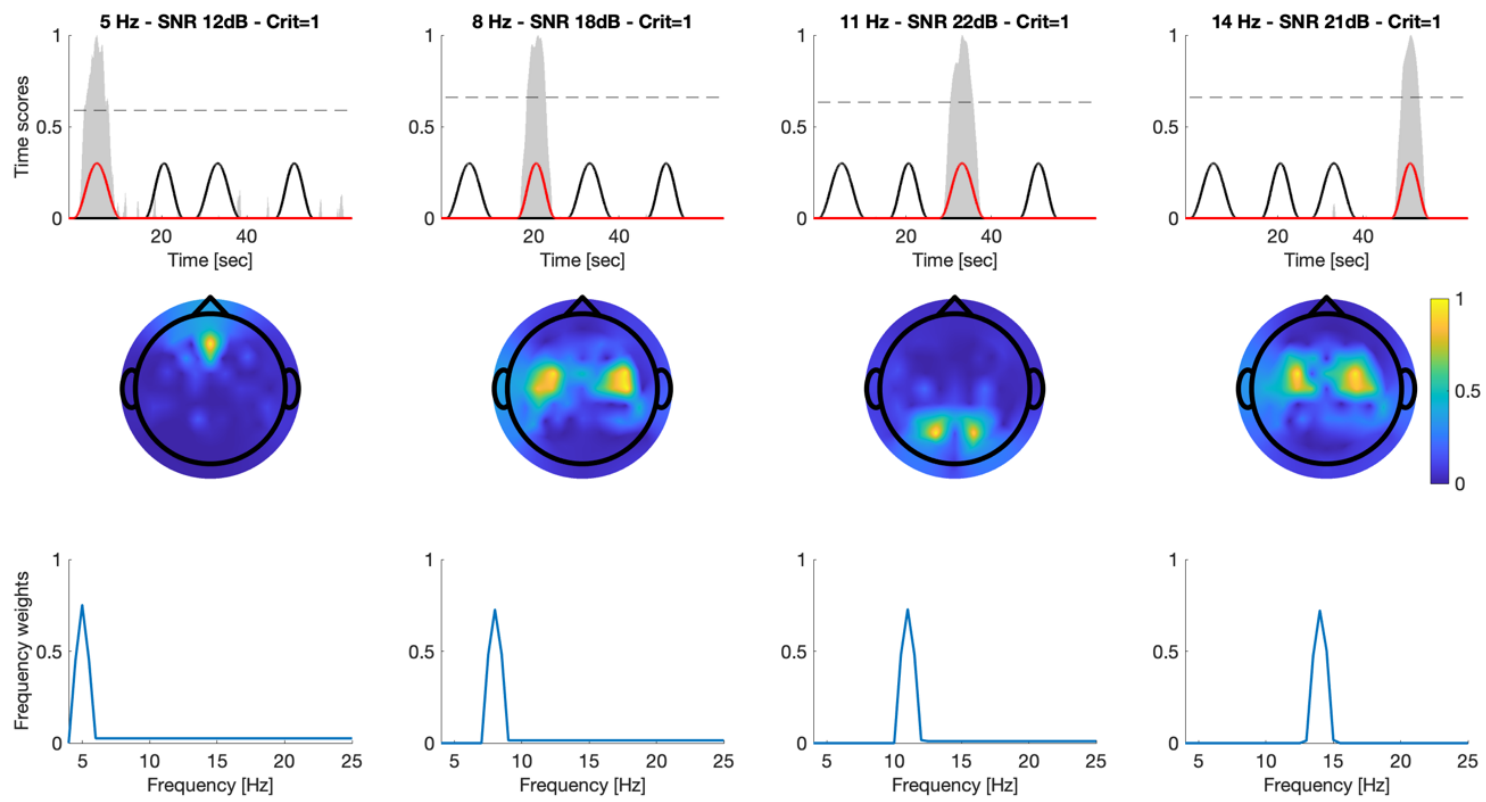
Figure 6: PARAFAC results. Description of plots same as in Figure 4. A) PARAFAC for SNR $R_{\text {low }}$ applied to the EEG dataset. B) PARAFAC for SNR low applied to the SSL-EEG dataset.

A)
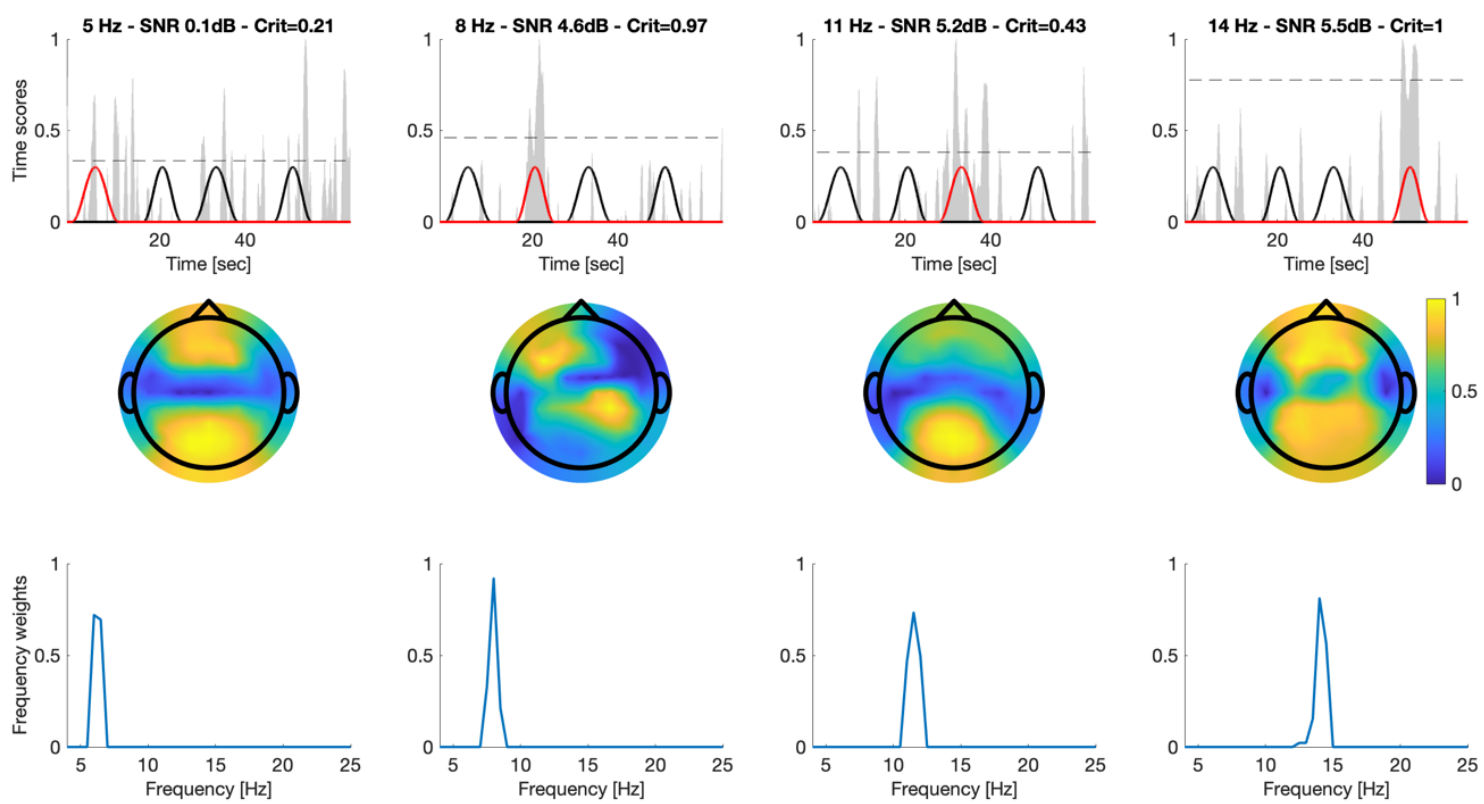

B)
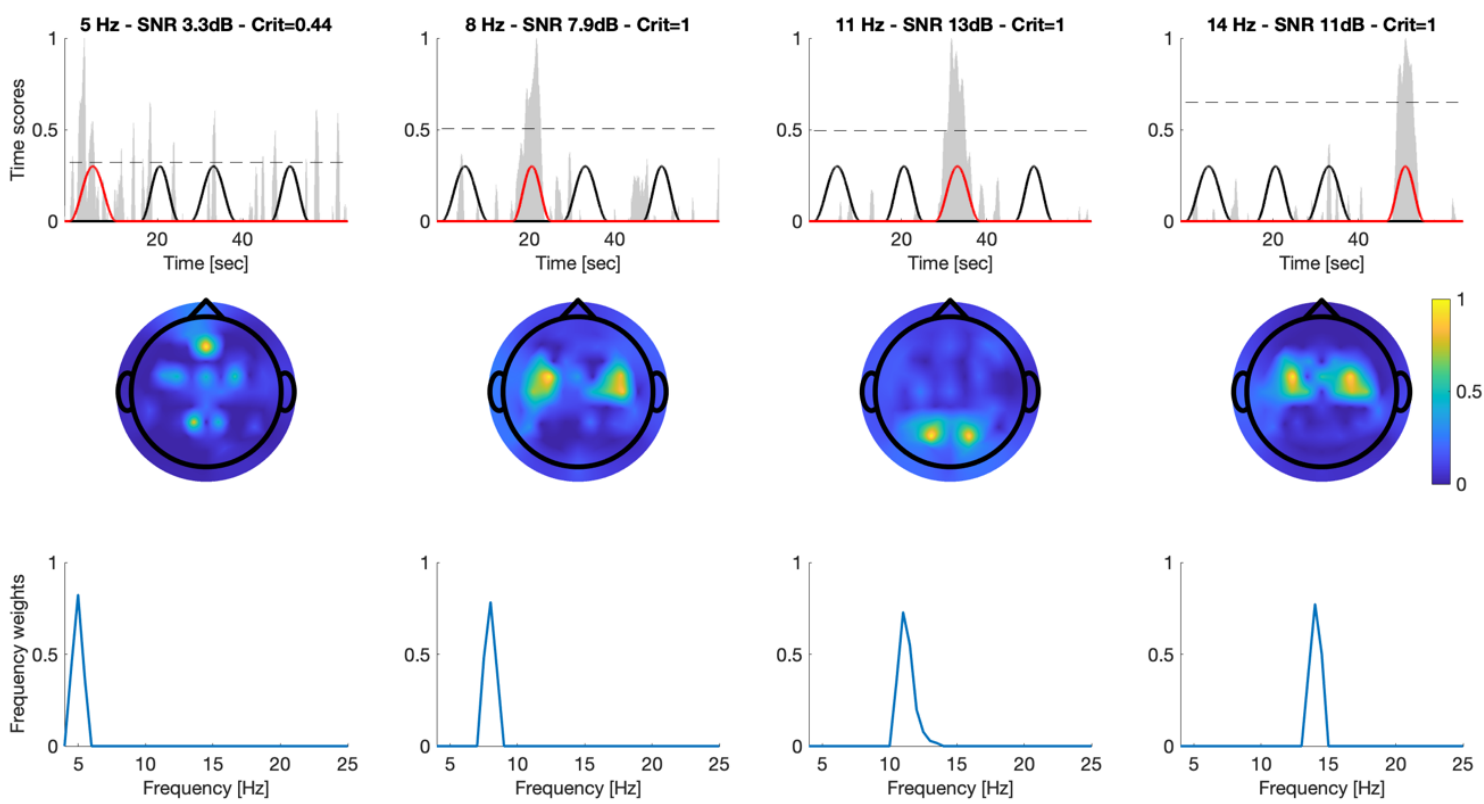
Figure 7: PARAFAC with a bimodality constraint on spatial signatures (SS). Nonnegativity constraint on SS is applied to all solutions. PARAFAC was applied to the SSL-EEG dataset with SNRlow. The columns represent: 1) the solution without bimodality constrain 2) the solution with bimodality constraint 3) the solution with bimodality constraint and the midline and left hemisphere EEG electrodes used 4) the solution with bimodality constraint and the midline and right hemisphere EEG electrodes used. A) Solution for $\mu$ (8 $\mathrm{Hz})$. B) Solution for $\beta(14 \mathrm{~Hz})$.

A)
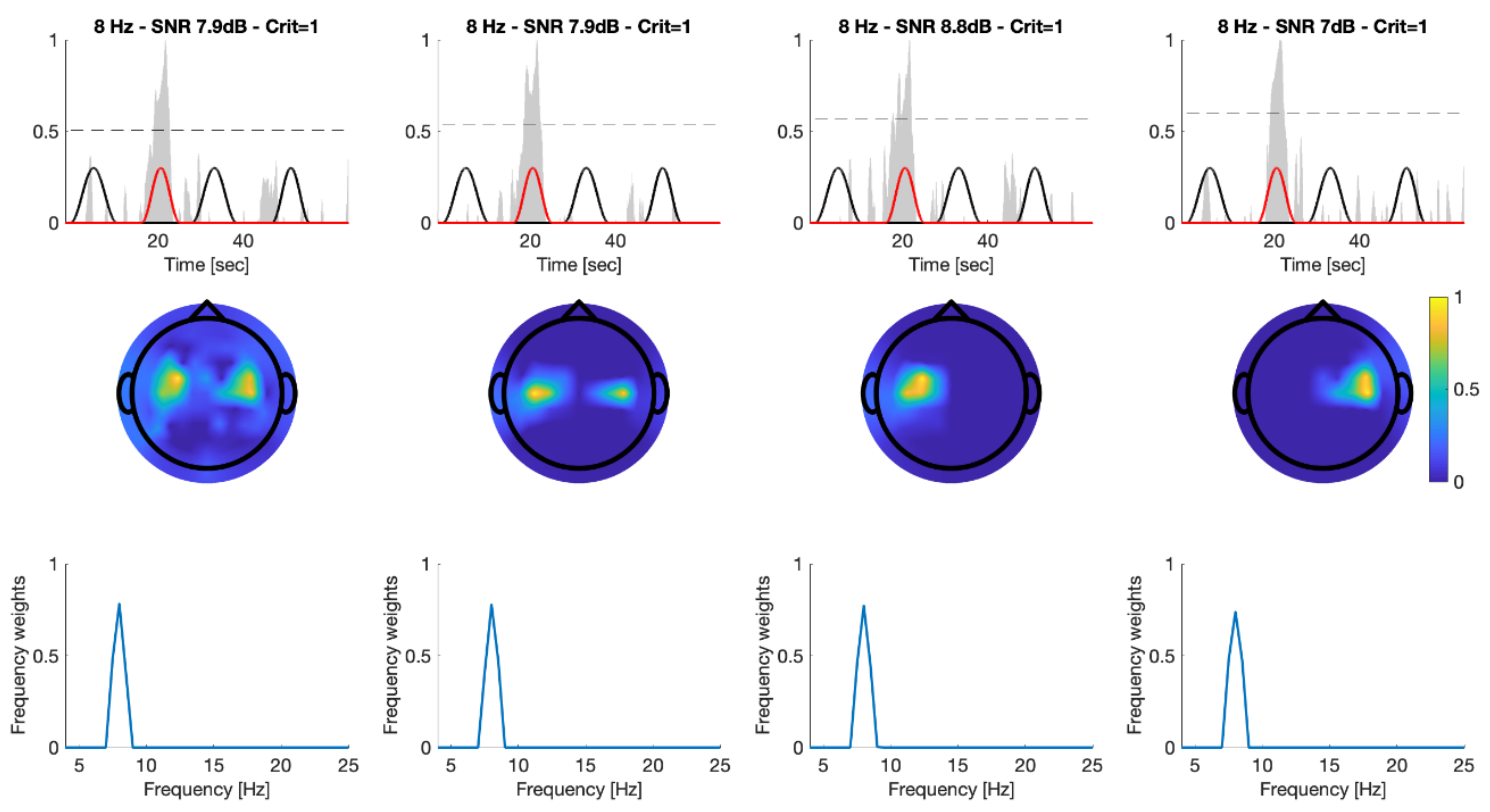

B)
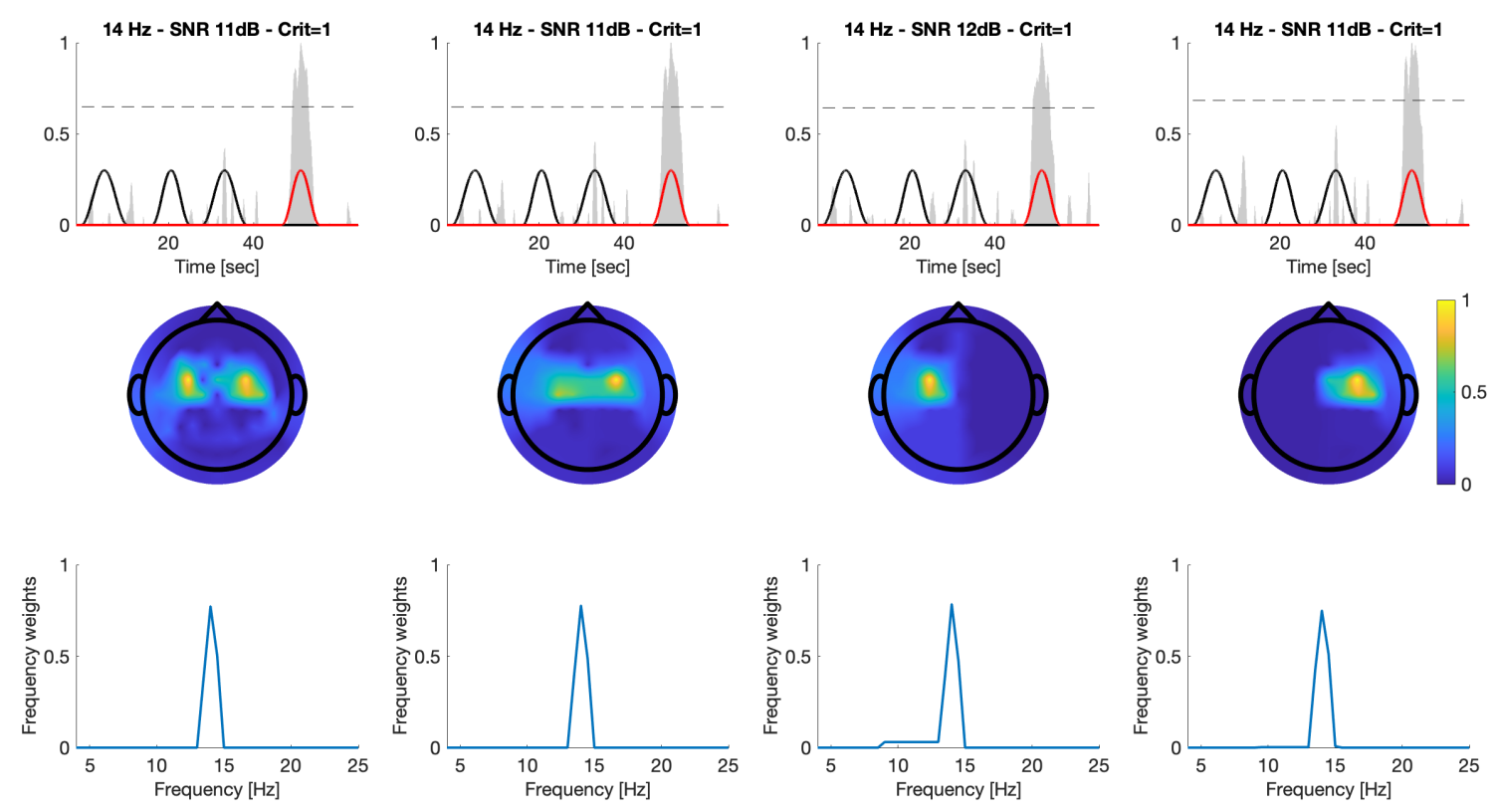
Figure 8: PARAFAC results of the SSL-EEG dataset with $S N R_{\text {low. }}$ A) A single activation periods B) Two activation periods.

A)
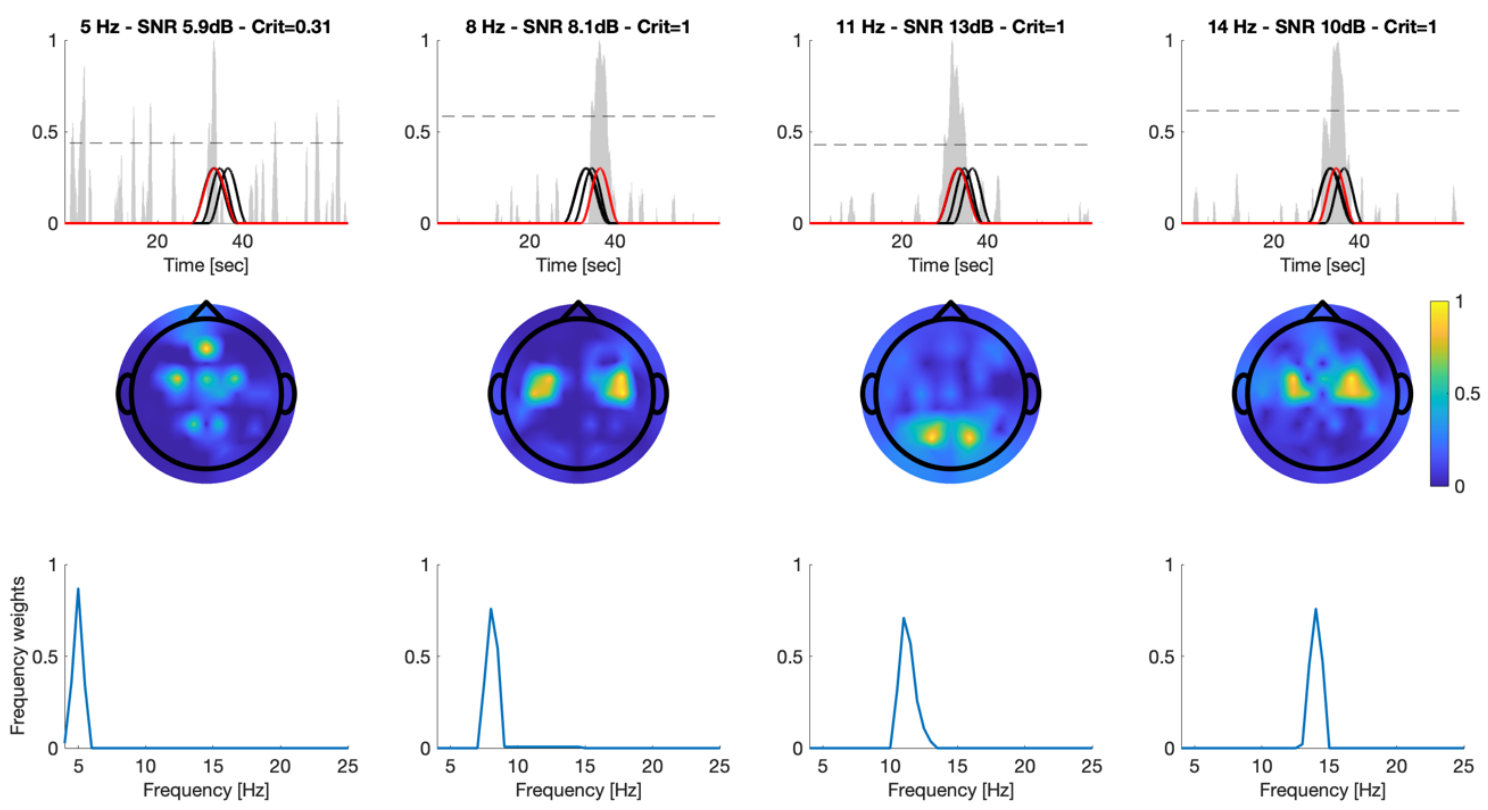

B)
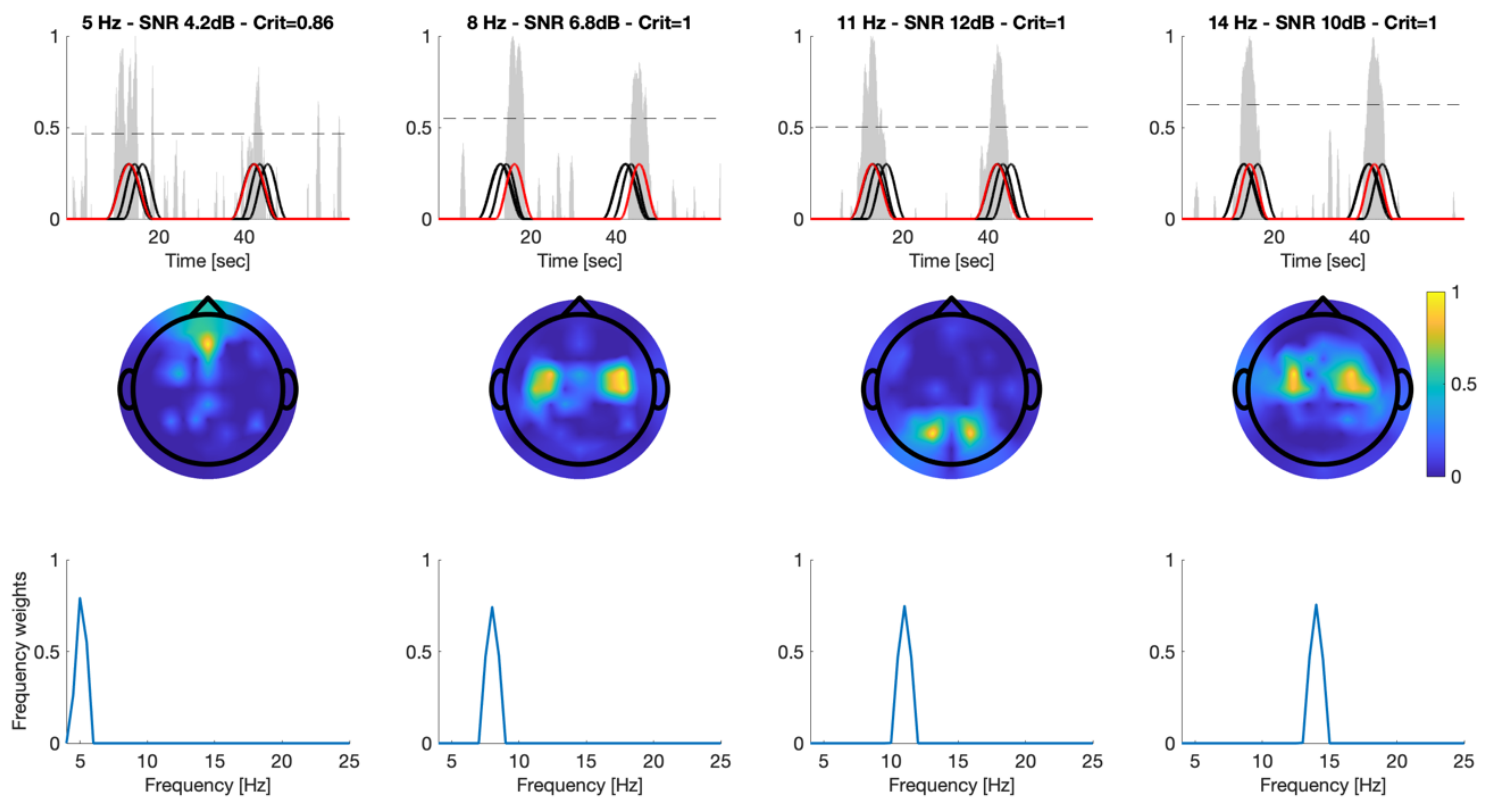
Figure 9: Time-scores after the projection of test data to the PARAFAC model depicted in Figure 6B. Top row: Time-scores of the EEG dataset with SNRlow. Second row: Time-scores of the SSL-EEG dataset with $S N R_{\text {low. }}$ Third and fourth row: spatial and frequency signatures of the training model depicted in Figure $6 \mathbf{B}$.
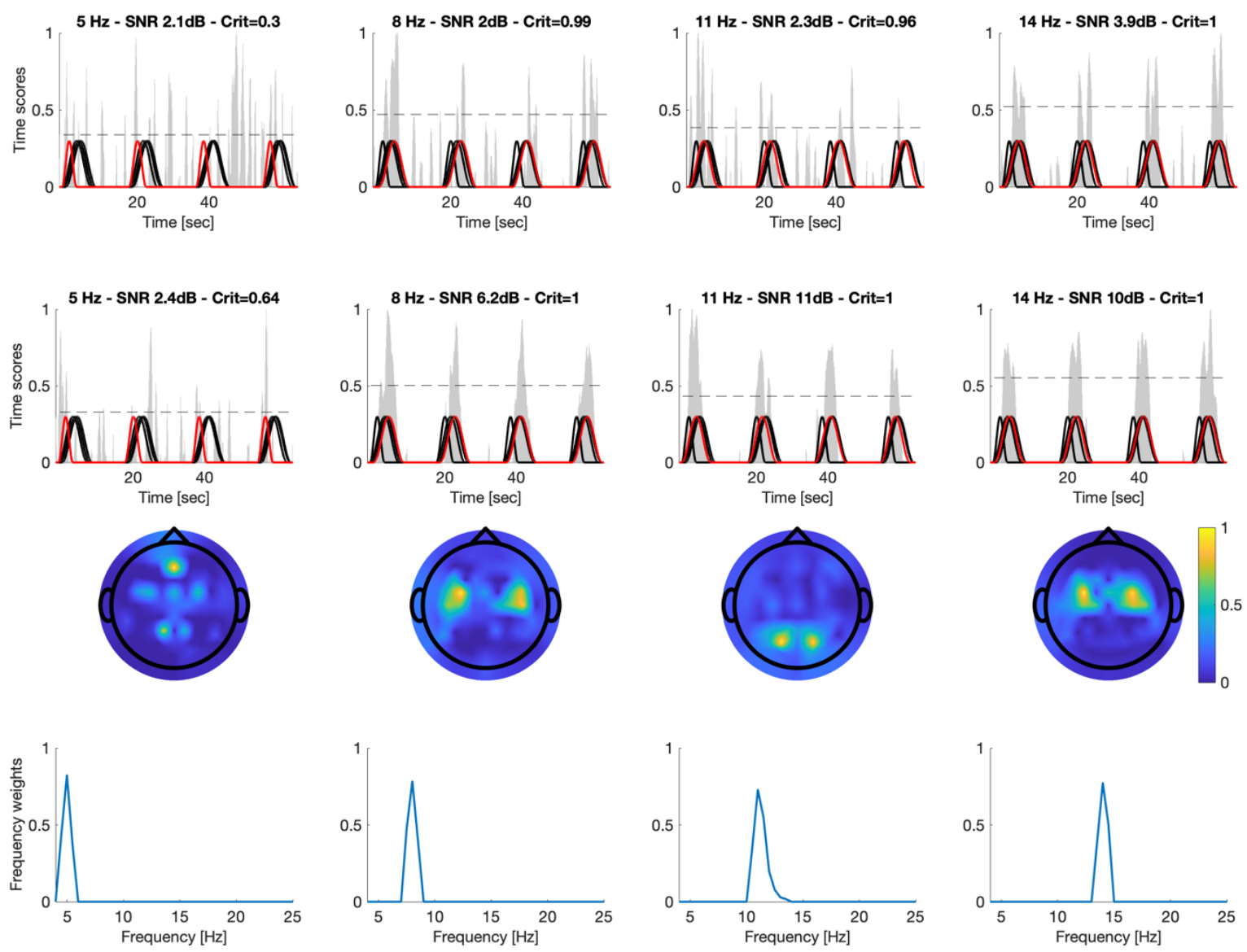
Figure 10: A) The (2,4,4)-NTD model applied to EEG dataset with SNR high and NBOs time activation similar to Figure 8B, see section 3.5. The model consists of two time-scores (TS), four primary spatial signatures (pSS), and four frequency signatures (FS). For each combination of time-scores (TS) (first column) and frequency signatures (FS) (first row), a topographic map of the corresponding spatial signature (SS) is plotted. The SSs represent a linear combination of pSS with weights representing elements of the mixing tensor rows corresponding to the spatial modality. B) Graphical representation of the mixing tensor $G \in$ $R_{+}^{2 \times 4 \times 4} . G(1,: ;)$ represents the first mode slice of $G$ associated with TS 1 , which was obtained by fixing the first mode index to 1 and varying the indices in the second and the third mode. Similar for $G(2,: ;)$.).

A)
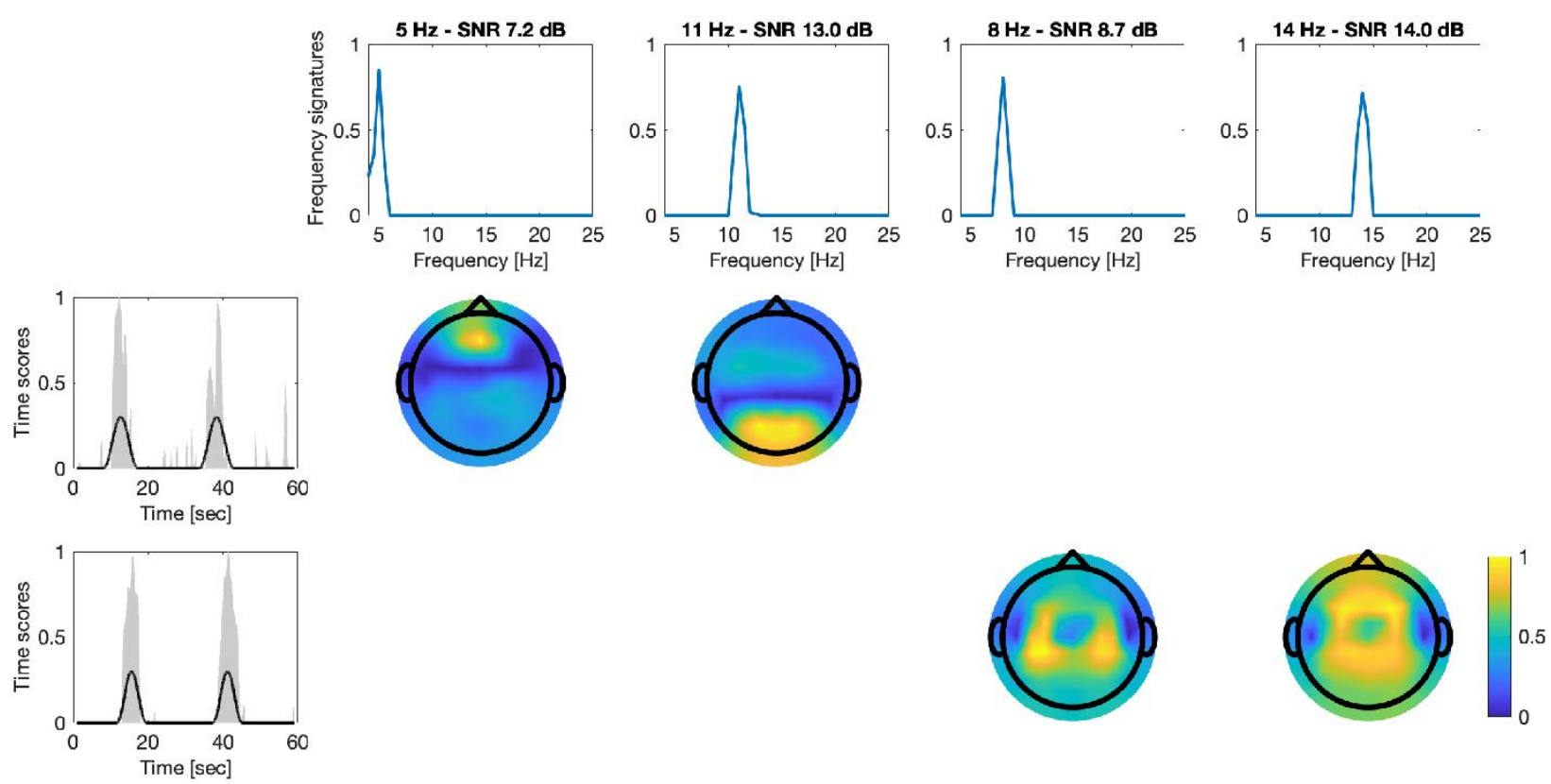

B)
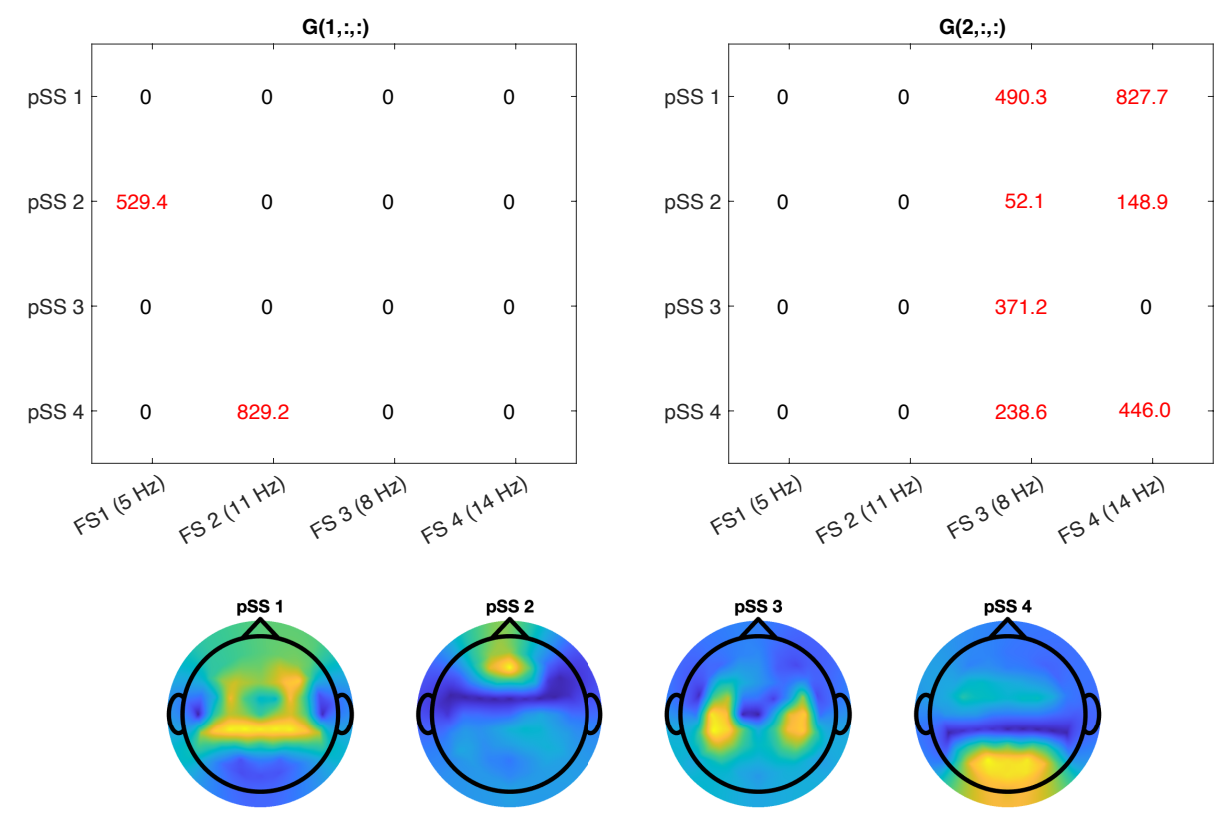
Figure 11: A) An example of an alpha rhythm burst followed by the periods of its absence. B) Topographic maps of the total and oscillatory power spectral parts at the $10.5 \mathrm{~Hz}$ frequency. Maps for raw and SSL transformed EEG are depicted. C) Total and oscillatory power spectrum of raw EEG computed at four selected EEG electrodes. The + marker specifies the $10.5 \mathrm{~Hz}$ frequency.

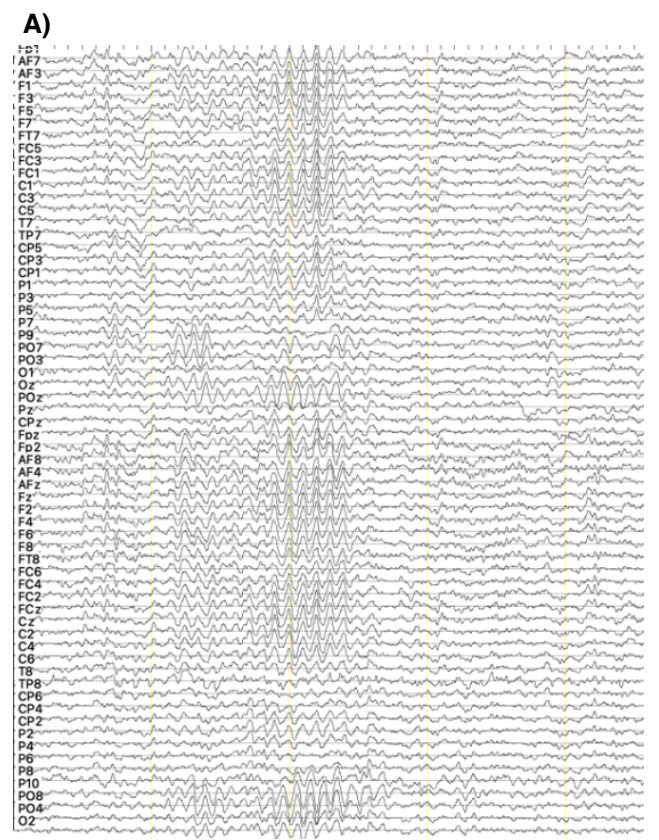

B)

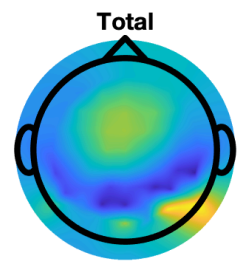

C)

$$
\text { PO7 }
$$

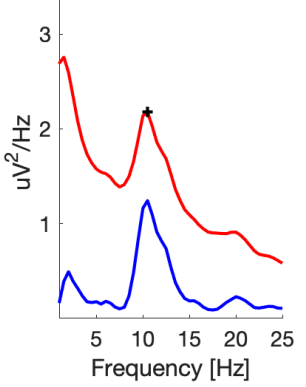

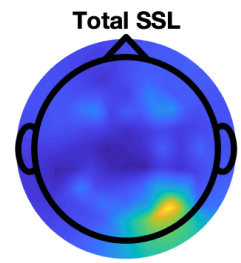

C3

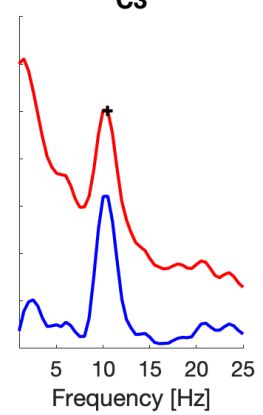

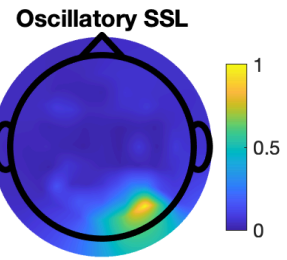

C4

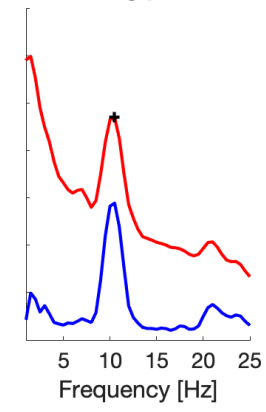


Figure 12: SSD and PARAFC results on real EEG. Each column represents absolute time-scores, spatial signature and frequency signature of each component. The frequency signature of the SSD components is represented by the oscillatory part of the corresponding time-scores power spectrum. Within the timescores (grey) plots, the power of the $10.5 \mathrm{~Hz}$ alpha rhythm is also depicted (black). The power was computed at the PO8 electrode for SSD component 1 atom PARAFAC atom 1, at PO7 for the component and atom 2. In the case of PARAFAC, each power value represents the power of the $10.5 \mathrm{~Hz}$ alpha rhythm averaged across a $2 \mathrm{sec}$ long window to match the PARAFAC time-scores. For illustrative purposes, the power values of the $10.5 \mathrm{~Hz}$ alpha were rescaled to the 0 to 0.25 interval; all other values, but the oscillatory power spectrum of SSD components, were rescaled to the 0 to 1 interval. A) Results on raw EEG. B) Results on SSL transformed EEG.

A)
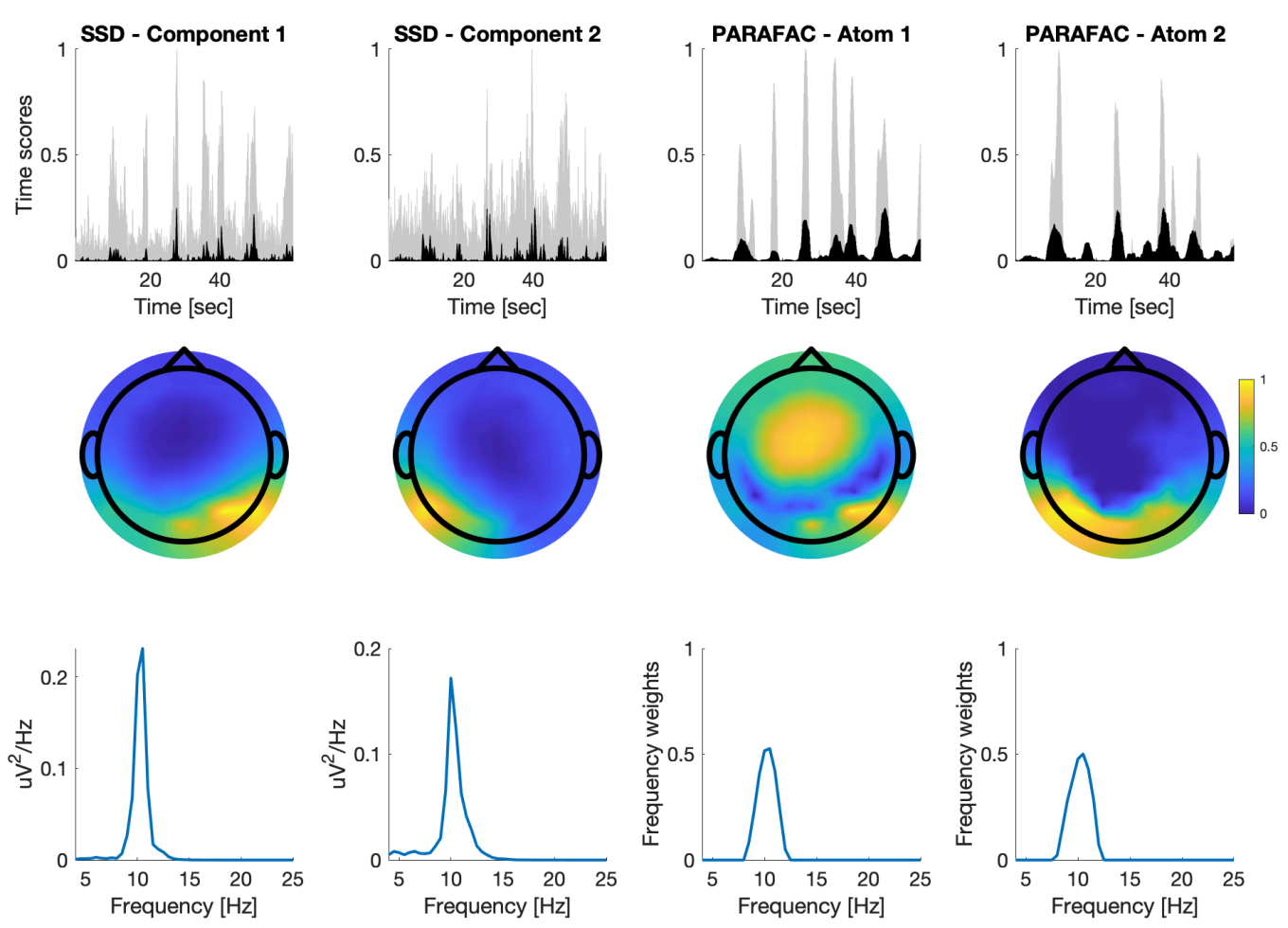
B)
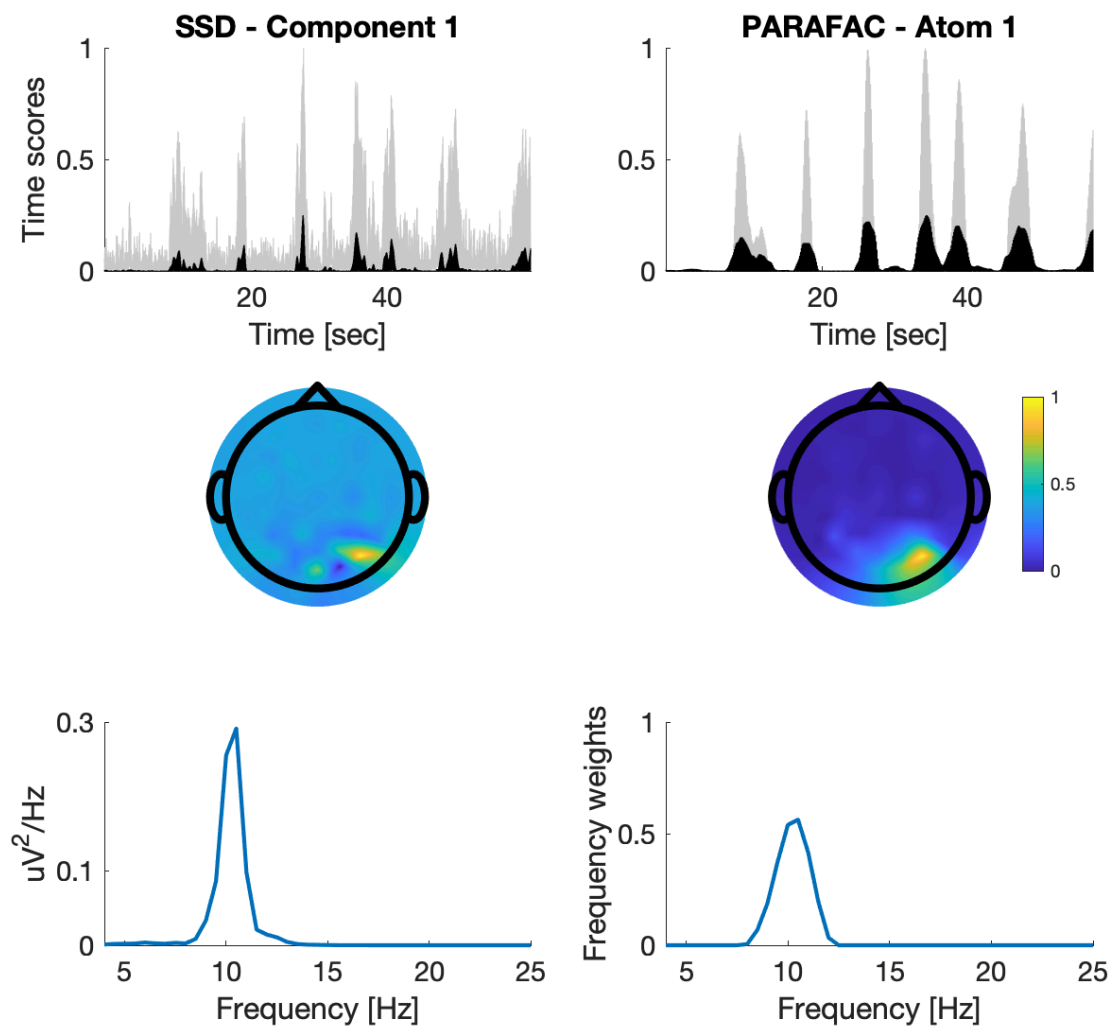
Table 1: Summary of the signal to noise ratios (SNR) values for all narrowband scalp-recorded EEG oscillations (NBOs) and the two levels of the added background brain activity (SNR high $_{\text {and }}$ SNR $R_{\text {low }}$ ). SSL-EEG represents scalp EEG records after surface spline Laplacian transformation. The values correspond to the generated data when the NBOs' time activation doesn't overlap.

\begin{tabular}{|c|c|c|c|c|c|c|}
\hline \multirow[b]{2}{*}{ NBO } & \multicolumn{3}{|c|}{ EEG } & \multicolumn{3}{|c|}{ SSL-EEG } \\
\hline & $\begin{array}{c}\text { EEG } \\
\text { electrode }\end{array}$ & SNR $R_{\text {high }}[\mathrm{dB}]$ & $\mathrm{SNR}_{\text {low }}[\mathrm{dB}]$ & $\begin{array}{c}\text { EEG } \\
\text { electrode }\end{array}$ & $\mathrm{SNR}_{\text {high }}[\mathrm{dB}]$ & $\mathrm{SNR}_{\text {low }}[\mathrm{dB}]$ \\
\hline midline $\theta-5 \mathrm{~Hz}$ & $\mathrm{AFz}$ & 7.2 & 0.1 & $\mathrm{AFz}$ & 12.2 & 3.3 \\
\hline left $\mu-8 \mathrm{~Hz}$ & $\mathrm{CP} 3$ & 13.4 & 4.9 & FC3 & 17.3 & 8.8 \\
\hline right $\mu-8 \mathrm{~Hz}$ & CP4 & 12.9 & 4.3 & FC6 & 17.8 & 7.0 \\
\hline left $\alpha-11 \mathrm{~Hz}$ & PO3 & 15.2 & 5.2 & PO3 & 22.2 & 13.6 \\
\hline right $\alpha-11 \mathrm{~Hz}$ & $\mathrm{PO} 4$ & 14.3 & 5.2 & PO4 & 22.2 & 13.3 \\
\hline left $\beta-14 \mathrm{~Hz}$ & F3 & 15.1 & 5.7 & FC3 & 19.9 & 11.6 \\
\hline right $\beta-14 \mathrm{~Hz}$ & F4 & 15.1 & 5.3 & FC4 & 21.2 & 10.1 \\
\hline
\end{tabular}

